Document de Recherche du Laboratoire d'Économie d'Orléans DR LEO 2017-13

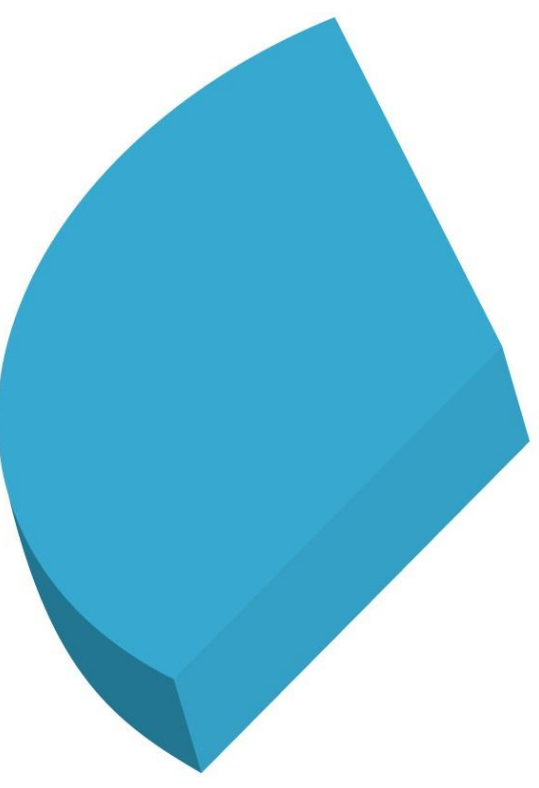

Du MEDAF avec risque Systémique à la détermination des Institutions

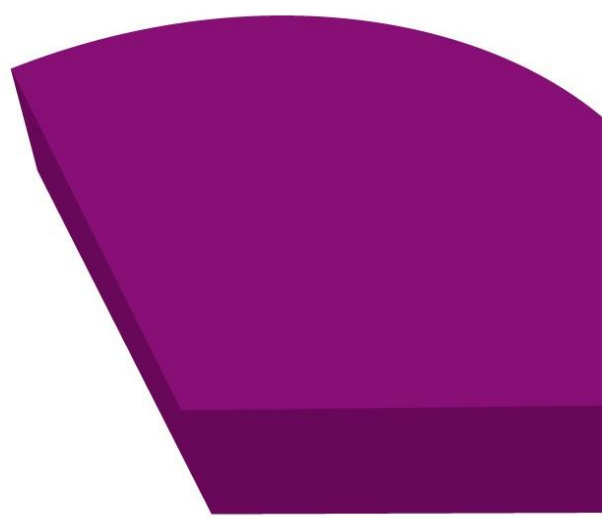
Financières d'Importance Systémique

Jean-Charles GARIBAL

Patrick KOUONTCHOU

Bertrand MAILLET

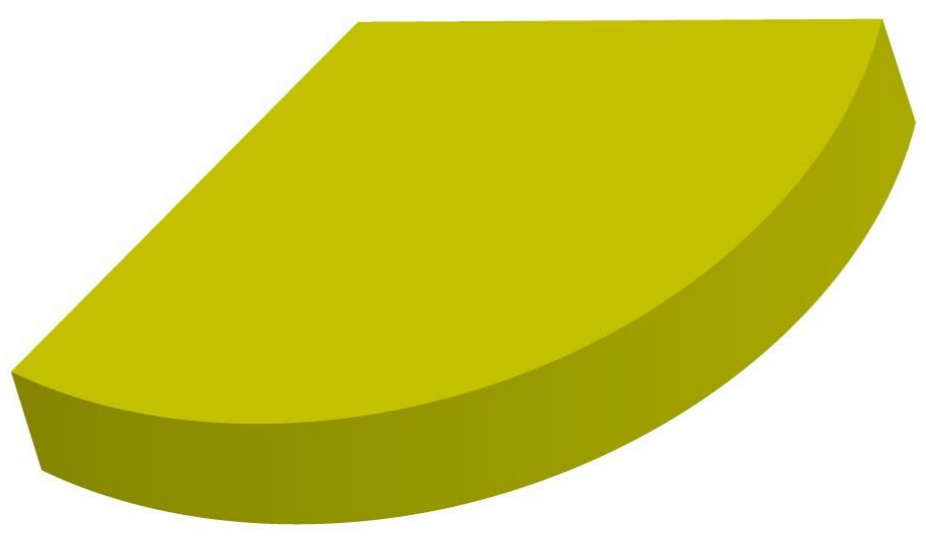

Mise en ligne : 06/11/2017

A paraître dans la Revue Economique 


\title{
Du MEDAF avec risque Systémique à la détermination des Institutions Financières d'Importance Systémique
}

\author{
Jean-Charles Garibal $^{\phi}$, Patrick Kouontchou ${ }^{\delta}$, Bertrand Maillet ${ }^{\gamma}$
}

Octobre 2017

\begin{abstract}
Nous nous proposons de tester dans cet article une extension du MEDAF, dans lequel nous ajoutons un facteur de risque systémique, mesuré par une agrégation des principales mesures de risques systémiques, comme proposée récemment par différents auteurs, dans le cadre d'une Analyse en Composantes Principales Parcimonieuse (ACPP). Il ressort de nos analyses empiriques menées sur le marché américain que le risque systémique est effectivement une composante importante de la rémunération sur certains titres. Nous proposons finalement une application originale servant à l'identification et au classement des Institutions Financières d'Importance Systémique (IFIS).
\end{abstract}

\section{From the CAPM with Systemic risk to the designation of the Systemically Important Financial Institutions}

We propose herein to test an extension of the traditional CAPM, in which is added a factor of systemic risk, build thanks to a Sparse Principal Component Analysis (SPCA) of a large set of systemic risk measures, as recently proposed by several authors. Empirical tests show that, on the American equities market, the new systemic risk factor is highly significant when pricing assets. At the end, we propose an original application in order to detect and rank Systemically Important Financial Institutions (SIFI).

JEL codes: C45, C53, C58, G01, G11.

Keywords: CAPM, Systemic Risk, SIFI, SPCA.

\footnotetext{
\$ Univ. Orléans, CNRS, LEO, UMR 7322, F-45067, Orléans, France. Courriel : jean-charles.garibal@univ-orleans.fr.

${ }^{\delta}$ Univ.Lorraine (CEREFIGE). Correspondance : Faculté de droit, économie et administration, Île du Saulcy, 57045, Metz Cedex 01. Courriel : patrick.kouontchou@univ-lorraine.fr.

$\gamma$ EMLyon (CEFRA) et Variances. Correspondance : EMLyon Business School - Campus de Paris, 15 boulevard Diderot, 75012 Paris. Courriel : bmaillet@em-lyon.com

Nous remercions ici Sylvain Benoit, Régis Breton, Laurent Clerc, Olivier De Bandt, Fany Declerck, Christophe Hurlin, Catherine Lubochinsky, Joël Petey, Roger Stein, Bertrand Tavin, Sessi Tokpavi et AnneGaël Vaubourg pour leurs commentaires, leurs retours positifs et leurs encouragements, ainsi que les participants aux conférences de l'AFFI (Liège, 2016), FEBS (Malaga, 2016), GDRE (Clermont-Ferrand, 2016), IRMC (Jérusalem, 2016) et IRIAF (Niort, 2016). Enfin, les auteurs remercient le GRI in Financial Services (www.globalriskinstitute.org) pour son soutien. Des ressources en lien avec cet article sont disponibles sur : www.systemic-risk-hub.org. L'avertissement habituel s'applique.
} 


\section{Introduction}

La récente crise financière de 2008 s'est distinguée à la fois par la rapidité de l'effet de contagion financière et aussi par son impact très négatif sur le secteur réel - avec pour conséquence une contraction de l'activité économique dans bon nombre de pays développés. Une des problématiques majeures en économie financière à la suite de ces turbulences sur les marchés financiers a été, en premier lieu, de tenter de s’accorder sur une (ou des) définition(s) du risque systémique, multiforme par nature, en insistant sur telle ou telle caractéristique primordiale de celui-ci. Il s’agissait ainsi de mettre en avant les différents aspects de ce risque : taille de l'institution financière en état de choc, effet de levier et risque extrême de marché, pénurie de liquidité, phénomène d’interconnexions entre les acteurs et de contagion des chocs, ont ainsi été identifiés comme les principaux éléments constitutifs d'une crise systémique. Il s’agissait aussi, en second lieu, de construire des outils pertinents d'analyse pour quantifier le risque systémique, et de nombreux auteurs ont ainsi proposé des mesures rendant compte tant de l'état général du système que de l'identification des principales institutions contribuant à ce risque global ${ }^{1}$. La littérature académique a ainsi accompagné ce nouvel impératif en matière de supervision bancaire, en proposant un certain nombre de métriques ou mesures de risque systémique $^{2}$. Sont ainsi distinguées d'un côté les mesures individuelles qui évaluent le risque systémique des institutions prises isolément, et, de l'autre, celles qui ont pour objectif de mesurer le risque systémique global ${ }^{3}$.

Mais, si effectivement plusieurs mesures ont ainsi déjà été proposées pour quantifier le risque systémique des institutions financières, chacune insiste de fait sur tel ou tel aspect particulièrement évident du risque systémique. Deux approches peuvent être ici distinguées. L’une est fondée sur l’information publique, qui a le mérite d'être relativement transparente et qui permet d'obtenir des mesures disponibles quasiment en

\footnotetext{
${ }^{1}$ Un site internet dédié aux classements des IFIS (depuis 2011) est disponible à l'adresse : www.sifiAtch.fr.

${ }^{2}$ Un site internet dédié au risque systémique (avec une liste exhaustive des mesures existantes et des ressources en lien avec cet article) est disponible à l'adresse : www.systemic-risk-hub.org.

${ }^{3}$ Par souci d'économie de place dans cet article, nous renvoyons le lecteur intéressé à Kouontchou et al. [2017] et une annexe complémentaire (dédiée en outre à la définition des mesures de risque systémique utilisées dans cet article) est disponible auprès des auteurs sur demande. Pour une revue complète de la littérature sur les mesures de risque systémique, voir par exemple Bisias et al. [2012] ; De Bandt et al. [2015] et Benoit et al. [2017a]. Voir aussi, plus spécifiquement, Abergel et al. [2013] ; Diebold et Yilmaz [2014]; Hautsch et al. [2015] et Billio et al. [2017] pour la littérature sur les réseaux servant à quantifier le risque systémique.
} 
temps réel. L’autre approche, plus complète, repose aussi sur des informations privées complémentaires (comptables et/ou réglementaires). La première approche a concentré l'essentiel des efforts de recherche et plusieurs voix s'élèvent aujourd'hui pour pointer les limites et les incertitudes de ce type de mesures. Rien de plus dangereux en effet que de construire une régulation sur un ensemble de critères peu fiables menant à une pseudosécurité du système financier, qui serait de facto mise à mal dès que le risque se matérialiserait effectivement. Rien de plus injuste aussi qu'un système qui reposerait sur de mauvais critères : les acteurs sanctionnés paieraient pour d'autres.

Ainsi, afin de limiter le risque de modèle lié à l'estimation des différentes mesures de risque systémique (e.g. Daníelsson et al. [2015] ; Boucher et al. [2016]), plusieurs propositions concernant la construction d'un indice de risque systémique viennent d'être avancées, notamment en utilisant des techniques de réduction de l'information (Nucera et al. [2013] ; Giglio et al. [2016] ; Kouontchou et al. [2017]).

C'est dans ce contexte que nous proposons dans cet article une méthodologie claire et transparente qui permettrait d'identifier les Sociétés Financières d'Importance Systémique (IFIS, adapté de l'anglais Systemically Important Financial Institutions SIFI).

La deuxième limite des mesures de risque systémique est liée à leur validation empirique, c'est-à-dire à notre capacité à proposer un critère formel permettant de juger la pertinence de l'approche. Plusieurs classements d'IFIS coexistent en effet, sans qu'il n’existe de véritable critère statistique ou économique permettant de privilégier l'un ou l'autre de ces classements à l'aune d'un critère objectif unanimement admis. Certains résultats tendent déjà par ailleurs à montrer que les principales mesures de risque systémique proposées sont relativement peu fiables (Kupiec et Guntay [2015]). Outre leur fragilité statistique, de nombreuses firmes non-bancaires sont signalées comme systémiques par ces mesures et les résultats apparaissent sensibles à la mesure retenue. Toutefois, plusieurs initiatives ont émergé récemment, visant à proposer des critères de validation économique (Giglio et al. [2016] ; Kouontchou et al. [2017]). C'est aussi dans ce courant de littérature que notre article s'inscrit.

Une fois le niveau global du risque systémique mesuré, l'objectif du présent article est de proposer un cadre théorique simple pour illustrer plusieurs canaux par lesquels le risque systémique pourrait affecter les prix des actifs. En effet, le risque systémique, vu 
comme une caractérisation extrême du risque de marché, semble intuitivement jouer un rôle dans l'évaluation des prix des actifs, bien que ce rôle soit difficilement identifié du fait de l'existence supposée d'une relation probablement non-linéaire qui lie le risque systémique aux prix des actifs. Il est ainsi souvent noté que les turbulences de marché, passées un certain seuil, s'accompagnent la plupart du temps de pertes significatives sur les marchés financiers. Le rôle des interconnexions entre les institutions a aussi été mis en évidence depuis la crise de 2008-2009. Nous essayons ainsi dans la suite de montrer comment le risque systémique modifie la relation d'équilibre du Modèle d'Evaluation Des Actifs Financiers (MEDAF, de l'anglais Capital Asset Pricing Model - CAPM ; Cf. Sharpe [1964]), et l'estimation des primes de risque liées à chaque facteur. Nous verrons ainsi, dans le cadre de ce modèle d'évaluation, qu'un portefeuille avec un risque systématique élevé pourrait en fait contenir un risque systémique non négligeable. Enfin, nous envisagerons d'utiliser les résultats de l'estimation de modèle d'évaluation étendu au risque systémique, pour évaluer la sensibilité (et la dangerosité pour le système global) des différentes institutions financières. Plus précisément, notre méthodologie procède en trois étapes comme expliqué dans la suite.

Dans la première étape, nous retenons l'Analyse en Composantes Principales dite Parcimonieuse (ACPP) comme outil de construction d'un indice agrégé, à la suite de Kouontchou et al. [2017]. Cette méthode de réduction de dimension a la particularité de sélectionner un certain nombre de composantes actives dans l’indice global agrégé. Dans le cas présent, elle a l'avantage d'écarter, lors de la construction de l’indice agrégé de risque, les mesures qui n'apportent que peu à l'explication globale de l'hétérogénéité des données. L'ACPP a aussi l'avantage de permettre l'endogénéisation du paramètre du nombre de mesures à retenir dans la construction d'un indice global représentatif. La valeur optimale de ce paramètre est ici obtenue en retenant l’indice agrégé dont les variations extrêmes causent le plus, au sens de Granger, les variations extrêmes de l’activité économique. L’inférence est réalisée en utilisant le test non paramétrique de causalité dans les risques extrêmes de Hong et al. [2009]. Cette approche nous permet de tester un nombre important de décalages temporels dans la relation causale, en escomptant la contribution des décalages les plus élevés. Nous poursuivons ainsi dans cette partie les travaux de Kouontchou et al. [2017] en élargissant encore le panel de mesures 
considérées, en augmentant le nombre d'institutions financières suivies et en étendant la période d'étude.

La deuxième étape de notre démarche concerne l'évaluation des actifs financiers. Il s'agit alors de savoir si le risque systémique, relatif dans une certaine mesure à chacun des titres, ne donne pas lieu à l'existence d'une prime de risque systémique, qui aurait été oubliée dans le cadre de la valorisation traditionnelle des actions. Le MEDAF en effet repose sur la définition de deux paramètres (Cf. Sharpe [1964], Lintner [1965] et Mossin [1966]), et il est considéré comme le principal modèle de référence pour l'évaluation des actifs financiers et des primes liées aux facteurs de risque. Cependant, l'expérience récente des crises financières a mis en exergue le risque que le système financier dans son ensemble ne s'effondre. Intuitivement, il nous semble que l'existence de chocs systémiques potentiels, particulièrement marqués pour les institutions financières lors de la dernière crise, devrait avoir un impact sur les prix des actifs financiers et qu'il devrait permettre de différencier les titres. Autrement dit, détenir en portefeuille des titres d'institutions particulièrement systémiques (les IFIS) devraient conduire à la révélation d'une prime spécifique liée au risque systémique. Toutefois, il existe déjà une vaste littérature sur de potentiels facteurs de risque intervenant dans la détermination des rentabilités boursières (Cf. Cochrane [2011] ; Holderness et Pontiff [2016] ; Harvey et al. [2016] ; Feng et al. [2017]). Tellement nombreux sont les facteurs que certains auteurs parlent d'un véritable «zoo » (Cochrane [2011] ; Harvey et al., [2016]; Feng et al. [2017]), ayant pour pensionnaires un grand nombre " d'animaux » - dont certains exotiques, pour imager la présence de nombreux facteurs évoqués dans les études. Dans le cadre de cette littérature et avec toutes les précautions envisageables ${ }^{4}$, il nous semble désormais important d'envisager l'étude de l'impact du risque systémique en termes de valorisation des actifs financiers, à l’instar par exemple des travaux de Billio et al. [2017]. Il ressort ainsi de nos analyses empiriques menées sur le marché américain que le risque systémique est effectivement une composante importante de la rémunération liée à certains titres.

La troisième et dernière étape de notre démarche concerne, la détection des IFIS. L'intuition est ici que, en utilisant le modèle d'évaluation proposé à la précédente étape,

\footnotetext{
${ }^{4}$ Notamment en termes de fixation des seuils de significativité. Harvey et al. [2016] par exemple indiquent qu'au seuil usuel de significativité de $5 \%$, les statistiques de Student devraient être supérieures à 3 pour que les différents facteurs soient jugés pertinents (voir aussi Feng et al. [2017]).
} 
il devrait être aisé d'isoler et de classer les institutions qui participent le plus au risque du système, en fonction de leur sensibilité (leur contribution) au risque global enregistré. En identifiant ces institutions financières, et en les mettant éventuellement «soussurveillance », les autorités de régulation pourraient ainsi, de manière transparente, mettre l'accent sur les faiblesses constatées du système et sur ses failles éventuelles (en s'attaquant systématiquement, et prioritairement, à la surveillance des institutions les plus systémiques).

La suite de cet article est organisée comme suit. Dans une première partie, nous présentons brièvement la méthode de construction de l'indice de risque systémique avec une illustration sur le marché américain. Après l'obtention d'un tel indice, nous présentons dans la deuxième partie le MEDAF et sa principale extension (celle de Fama et French [1992], [1993]), puis nous proposons le Modèle d'Evaluation des Actifs Financiers avec risque Systémique (MEDAFS). Nous rapportons ensuite nos principaux résultats empiriques d'estimation obtenus sur le marché américain. La troisième partie propose, enfin, une application originale du MEDAFS dans le cadre de la détection et du classement des institutions financières à caractère systémique (IFIS). La dernière partie conclut.

\section{De l'indice de risque systémique}

Pour intégrer le risque systémique comme facteur supplémentaire au même titre que le risque systématique ou le risque spécifique, il est nécessaire d'utiliser une mesure précise de ce risque extrême. Ces dernières années, s'est fortement développée une littérature sur l'identification des IFIS par des mesures quantitatives qui visent à caractériser le lien conditionnel entre les différentes institutions financières et le marché dans son ensemble. Toutefois, compte tenu des nombreuses dimensions du risque systémique, ces mesures individuelles ne permettent pas de détecter, complètement et de manière systématique, les institutions potentiellement systémiques.

Ainsi, l'utilisation de l'analyse factorielle comme outil d'agrégation de l'information issue de chaque mesure de risque systémique est une approche nouvelle. Moreno et Peña [2013] utilisent une ACP sur un ensemble de mesures individuelles de risque 
d'entreprises, pour obtenir un indice de risque systémique. Giglio et al. [2016] reprennent parallèlement cette démarche pour construire un indice de risque systémique et tester son pouvoir prédictif des futurs chocs sur les variables macroéconomiques en utilisant une régression par quantile. Nucera et al. [2016] utilisent, eux aussi, une ACP, portant sur un ensemble de six mesures de risque systémique. Néanmoins, leur étude diffère de celle de Giglio et al. [2016] puisqu'ils appliquent l'ACP sur les classements de 113 entreprises du secteur financier à travers un ensemble de mesures de risque systémique, et non sur un ensemble d'entreprises sur une période de temps donnée comme le font Giglio et al. [2016] dans leur étude effectuée à partir d'un ensemble de 19 mesures du risque systémique (au total). Kouontchou et al. [2017] poursuivent les travaux de Giglio et al. [2016] en utilisant une ACPP, sur la base de données utilisée par Diebold et Yilmaz [2009]. Nous reprenons, résumons et étendons dans la suite les travaux de Kouontchou et al. [2017] notamment en élargissant le nombre de mesures du risque systémique utilisées par Diebold et Yilmaz [2009] et en utilisant une extension actualisée de la base de données des institutions financières américaines étudiées par Brownlees et Engle [2017] et Kouontchou et al. [2017].

\section{Mesures spécifiques du risque systémique}

La littérature financière a ainsi proposé de nombreuses mesures quantitatives qui peuvent être utilisées pour l'identification des institutions potentiellement systémiques. Nous pouvons les regrouper en plusieurs catégories ${ }^{5}$.

Tout d'abord, des mesures de risque systémique global sont définies à partir de modèles économétriques de risque spécifiques à chacune des institutions. Il s’agit de la Conditional Value-at-Risk (CoVaR) et de la Delta Conditional Value-at-Risk ( $\triangle$ CoVaR) proposées par Adrian et Brunnermeier [2016], de la Marginal Expected Shortfall (MES) d'Acharya et al. [2013] et Brownlees et Engle [2017], de la SRISK d'Acharya et al. [2012] et Brownlees et Engle [2017] et de la mesure d'illiquidité d'Amihud [2002].

Ensuite, d'autres mesures se focalisent spécifiquement sur un aspect important du risque systémique, à savoir le niveau d'interconnexion des institutions financières ou de

\footnotetext{
${ }^{5}$ Par souci d'économie de place dans cet article, une annexe complémentaire dédiée à l'expression détaillée des mesures additionnelles à celles utilisées dans Kouontchou et al. [2017] est disponible sur demande auprès des auteurs.
} 
concentration du système financier ${ }^{6}$. Dans cette catégorie, nous sélectionnons le Spillover Index de Diebold et Yilmaz [2009], le Dynamic Causality Index (DCI) de Billio et al. [2012], la mesure de Turbulence de Kritzman et Li [2010], l'Absorption Ratio de Kritzman et al. [2011] et l'indice de concentration de Herfindahl-Hirschman.

Enfin, certaines variables macro-financières sont généralement utilisées comme des indicateurs avancés de l'activité économique (Cf. Estrella et Trubin [2006] ; Chen et al. [2009]). Nous en retenons ici respectivement : le Credit Default Yield Spread qui mesure l'écart entre le rendement des obligations corporate notées BAA et celle notées AAA par Moody’s ; Chen et al. [2009] montrent que cette variable est une mesure agrégée du risque de crédit robuste aux frictions (taxe et liquidité) sur le marché des obligations ; le TED Spread qui mesure l'écart entre le taux LIBOR à trois mois et les taux d'intérêts souverains à trois mois : une augmentation de cette variable est le signe que les prêteurs anticipent une augmentation du risque de crédit sur le marché des prêts interbancaires ; et enfin le Term Spread qui mesure la pente de la courbe des taux, et qui correspond à l'écart de rendement entre les bons du Trésor à dix ans et trois mois de maturité. Cette variable sert d'indicateur avancé de l'activité économique (Estrella et Trubin [2006]). Nous prenons en compte également la volatilité agrégée (Vol) et la Value-at-Risk (VaR) agrégée sur l'ensemble du système, pour tenir compte de l'évolution de la variabilité de ce dernier.

En comparaison avec les travaux de Giglio et al. [2016], Nucera et al. [2016] et Kouontchou et al. [2017] (qui utilisent respectivement 19, 6 et 12 mesures du risque systémique), 16 mesures de risque systémique sont utilisées dans cet article.

Ainsi, nous considérons 4 des 6 mesures utilisées par Nucera et al. [2016] : sont laissés de côté le Leverage ratio (LVG, défini dans Engle et al. [2014] comme étant la somme de la valeur de marché des capitaux propres augmenté des dettes et divisé par la valeur de marché des capitaux propres), et le Dollar Systematic Risk qui correspond au produit du bêta de la firme et de sa capitalisation boursière, car leurs caractéristiques sont déjà prises en compte dans les mesures SRISK, MES, CES et CoVaR. Par rapport à Giglio et al. [2016] qui utilisent 19 mesures au total (dont 9 originelles en comptant la volatilité agrégée et 4 variations de ces mesures, ainsi que 4 variables macro-économiques et 2 ratios de levier financier), nous laissons uniquement de côté : le Gilchrist-Zakrajsek spread (GZ) de Gilchrist et Zakrajsek [2012], la mesure CatFin de Allen et al. [2012] et

\footnotetext{
${ }^{6}$ Ibid., note 3, page 2.
} 
les agrégats Book Leverage et Market Leverage, car le comportement de ces mesures est proche de celles des variables, respectivement : Default Spread, VaR et SRISK. Ainsi aussi, nous complétons l'analyse de Kouontchou et al. [2017] établit sur un ensemble de 12 mesures de risque systémique, en prenant en considération en sus dans notre analyse : la SRISK de Brownlees et Engle [2017], la Value-at-Risk (VaR) agrégée, la volatilité agrégée et la Component Expected Shortfall (CES) de Banulescu et Dumitrescu [2015].

Dans la suite, nous commençons par illustrer la dynamique de nos différents indicateurs de risque systémique sur le graphique 1 suivant, construits à partir de notre base de données journalières relatives à des institutions financières américaines ${ }^{7}$ sur la période allant du 02/09/2003 au 26/02/2016. Nous constatons sur ce graphique une augmentation sensible de l'ensemble des mesures de risque systémique globales sur la période 2007-2008, qui correspond à la période de la crise financière. De même, bien qu’une tendance commune semble émerger de la dynamique des séries, il existe tout de même quelques disparités entre ces mesures. Ces différences peuvent provenir du fait que le risque systémique est multidimensionnel, chacune des différentes métriques en modélisant une dimension spécifique.

En effet, à partir de la matrice de leurs corrélations ${ }^{8}$, nous pouvons noter que toutes les corrélations sont statistiquement significatives au seuil de risque nominal de $5 \%$, à l'exception de la corrélation entre la mesure d’illiquidité et le TED Spread et entre le Dynamical Causality Index et le Herfindalh-Hirschman Index et entre le Dynamical Causality Index et le TED Spread pour les corrélations de Pearson.

\footnotetext{
${ }^{7}$ Par souci d’économie de place dans cet article, la liste exhaustive des Institutions Financières (IF) utilisées dans cet article est mise à disposition dans une annexe complémentaire disponible auprès des auteurs sur demande.

${ }^{8}$ Cette matrice est renseignée dans une annexe complémentaire disponible auprès des auteurs sur demande.
} 
Graphique 1 : Dynamique des seize mesures de risque systémique global
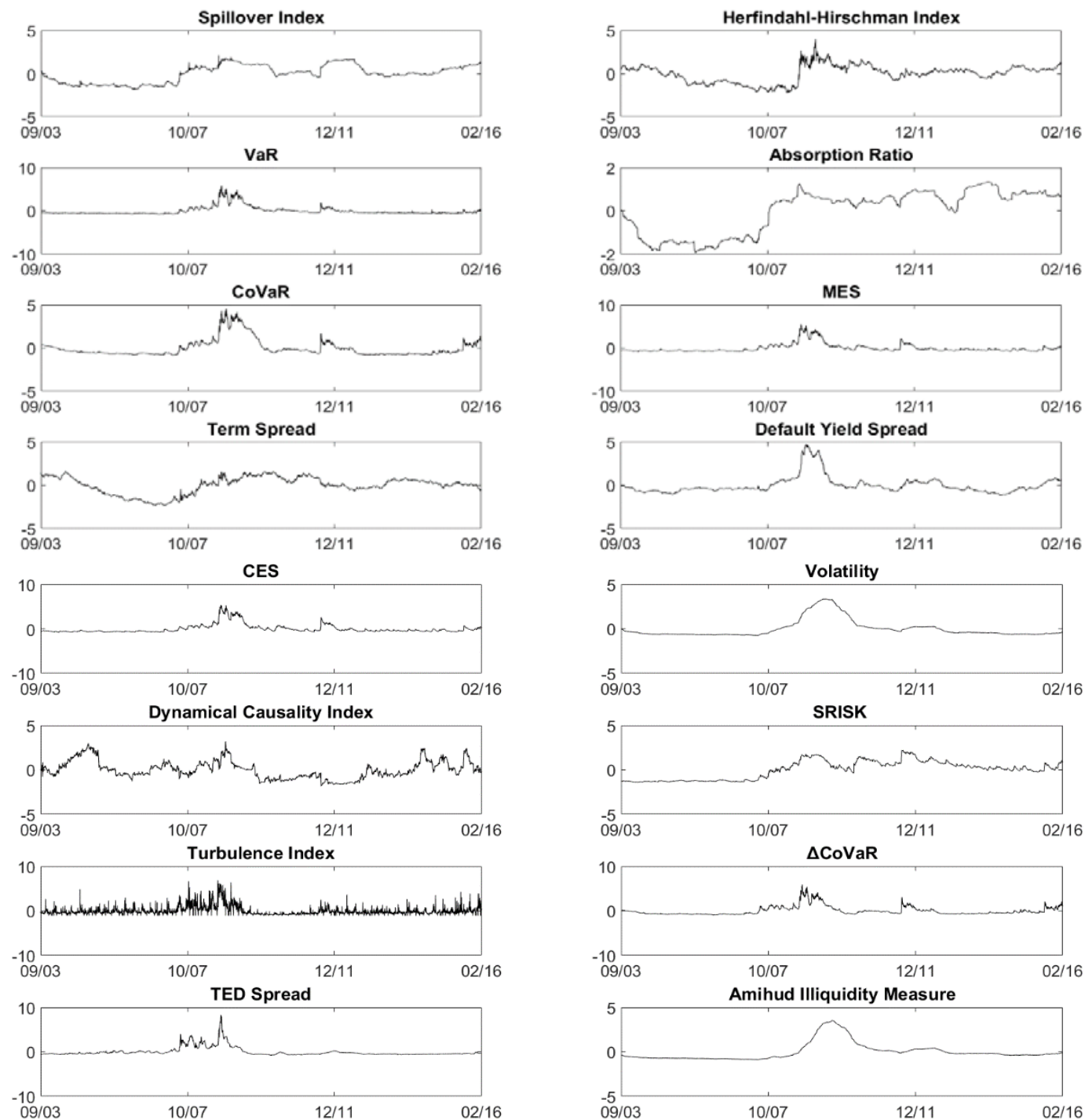

Source : Bloomberg, données journalières du 02/09/2003 au 26/02/2016 ; calculs des auteurs. Ces mesures, en dehors des variables macro-financières, sont estimées sur des fenêtres glissantes annuelles. Les séries brutes sont ici centrées sur leurs moyennes respectives et standardisées par leurs écart-types. Ces 16 mesures correspondent dans l'ordre à : $M_{1}$ : Spillover Index, $M_{2}$ : Herfindahl-Hirschman Index, $M_{3}:$ VaR, $M_{4}:$ Absorption Ratio, $M_{5}:$ CoVaR, $M_{6}:$ MES, $M_{7}$ : Term Spread, $M_{8}$ : Default Yield Spread, $M_{9}$ : CES, M10 : Volatility, M11 : Dynamical Causality Index, M12: SRISK, $M_{13}$ : Turbulence Index, $M_{14}: \triangle C O V a R, M_{15}:$ TED Spread et $M_{16}:$ Amihud Illiquidity Measure.

Les corrélations entre le TED Spread, la SRISK agrégée et la volatilité agrégée apparaissent également non significatives, ainsi que celles entre le Turbulence Index et la SRISK agrégée et entre le Dynamical Causality Index avec le Herfindahl-Hirschman Index et la $\triangle C o V a R$ agrégée. Les corrélations les plus fortes (supérieures à 0.90) sont celles relatives aux mesures de risque systémique global correspondantes à des moyennes de données en coupes instantanées de mesures individuelles (CoVaR, $\triangle \mathrm{CoVaR}, \mathrm{MES}$, 
$V a R)$. Il est donc nécessaire de proposer un indicateur qui intègre toutes les dimensions du risque (modèle agrégé) ${ }^{9}$.

Les mesures convoyant ainsi une quantité importante d'informations redondantes, puisqu'elles sont par nature des mesures d'un même phénomène, nous allons faire face à des problèmes liés à la multi-colinéarité des variables dans le cadre des régressions multiples qui sont bien connus : inflation de la variance des coefficients, absence de robustesse dans l'estimation des paramètres qui fluctuent de manière importante si l'échantillon change, signes des paramètres inversés par rapport à leurs valeurs théoriques prédites, variables importantes avec des coefficients faibles, impossibilité de déterminer l’importance relative des différentes variables, non-unicité des représentations estimées (e.g. Stewart [1987] et Green [2012]).

Il s’agira de prendre en compte ce phénomène, dans la prochaine sous-section, quand nous présentons plus précisément la construction de l’indice agrégé, basé sur l'ensemble des mesures de risque systémique.

\section{De l’indice agrégé de risque systémique}

Nous présentons brièvement dans cette section les résultats obtenus de la méthode construction de l'indice agrégé de risque systémique global ( $C f$. Kouontchou et al. [2017]). Dans un premier temps, nous utilisons une ACPP pour la réduction de dimension. Puis, dans un second temps, nous sélectionnons l'indice optimal de risque systémique via l'utilisation du test de causalité dans les risques extrêmes de Hong et al. [2009], afin de sélectionner l'indice optimal (parmi l'ensemble des indices possibles). ${ }^{10}$

L'ACP traditionnelle repose sur une décomposition d'un ensemble de variables sur la base de fonctions orthogonales qui sont déterminées à partir des données. Ces fonctions, qui sont des combinaisons linéaires des variables d’origine, visent à reproduire la variabilité existante dans les données. Elles correspondent aux axes (ou composantes principales) les plus importants. L’ACPP quant à elle, vise à obtenir une représentation similaire des données, en ne prenant en considération qu’un nombre limité de variables

\footnotetext{
${ }^{9}$ Ce dernier résultat est confirmé par l'analyse des corrélations conditionnelles entre les différentes mesures de risque. En effet, les corrélations au-dessus du quantile à $\alpha=80,00 \%$ (pics simultanés deux à deux des mesures de risque systémique) sont toutes égales à 1,00 et significatives au seuil de 1,00\%.

${ }^{10}$ Nous renvoyons aux articles de Hong et al. [2009] et de Kouontchou et al. [2017] pour plus de détails sur le test de causalité dans les risques extrêmes et la construction d'un indice optimal de risque systémique.
} 
(opération de sélection des variables). Nous suivons ici la méthodologie présentée dans Kouontchou et al. [2017] ${ }^{11}$.

Le tableau 1 suivant présente la composante principale dominante extraite de la méthodologie utilisant une ACPP avec les valeurs de la statistique de test de Hong et al. [2009] pour différentes valeurs du paramètre de lissage (appelé $\delta$; Cf. Kouontchou et al. [2017]). Ainsi pour $\delta=1,000$ correspondant à la contrainte la plus forte sur la norme 1 , le nombre de mesures de risque systémique global actives dans la composante dominante est égal à $k=1$. Il s'agit de la mesure $M_{1}$, à savoir le Spillover index de Diebold et Yilmaz [2009]. Lorsque $\delta$ augmente, la contrainte sur la norme 1 est moins contraignante, et des mesures additionnelles de risque systémique entrent dans la composante dominante. Pour illustration, lorsque $\delta=1,711$, quatre mesures sont actives dans l'indice, à savoir l'indice de concentration de Herfindahl-Hirschman, le Spillover index de Diebold et Yilmaz [2009], l’Absorption Ratio de Kritzman et al. [2011] et la Value-at-Risk (VaR) agrégée. Pour la valeur la plus élevée de $\delta$, toutes les mesures sont actives dans la composante dominante, et cette dernière correspond à la première composante issue d'une ACP classique. Notons que la dernière mesure de risque systémique à être activée dans une des composantes est la mesure d'illiquidité d'Amihud [2002]. Ce résultat n'est pas surprenant, dans la mesure où son comportement est très similaire à celui de la volatilité agrégée.

Usuellement, l'indice optimal sera jugé au sens d'un critère d'information classique (Cf. Hastie et al. [2015]). En lien avec les travaux de Giglio et al. [2016], Kouontchou et al. [2017] proposent plutôt d'utiliser le test non paramétrique de causalité dans les risques extrêmes de Hong et al. [2009] pour juger du meilleur des modèles. Ce critère permet de sélectionner l'indice qui aurait la statistique de Hong et al. [2009] la plus élevée. Autrement dit, il s'agit ici de choisir l'indice dont les variations extrêmes positives permettent d'expliquer au mieux les variations extrêmes négatives de l'activité économique.

\footnotetext{
${ }^{11}$ Nous renvoyons le lecteur intéressé à Kouontchou et al. [2017] pour la méthodologie utilisée, et une annexe complémentaire résumant l’approche est disponible auprès des auteurs sur demande.
} 
Tableau 1: Décomposition par variable des composantes principales parcimonieuses

\begin{tabular}{|c|c|c|c|c|c|c|c|c|c|c|c|c|c|c|c|c|}
\hline$\delta$ & & 30 & & 71 & 1,83 & 1,93 & 2,01 & 80 & - & 2,12 & 2,13 & & 2,24 & & 2,32 & \\
\hline$I d_{k}$ & & & & & & II & - & & & & & & & & , & \\
\hline$K$ & & 2 & 3 & $=4$ & $=5$ & $=6$ & $=7$ & $=8$ & $=9$ & $=10$ & $=11$ & & $k=13$ & & $k=15$ & \\
\hline$M_{1}$ & 00 & 92 & 01 & 83 & ,79 & ,75 & ,73 & ,73 & 77 & 0,70 & ,70 & 70 & 70 & 60 & 69 & 6 \\
\hline$M_{2}$ & 00 & 39 & 41 & ,45 &, 51 & ,53 &, 54 & ),54 & U,J4 & 0,5J & 0,55 & ענ, & טנ, & (ט) & 0,57 & 0,5 \\
\hline$M_{3}$ & 00 & 00 & 08 & 0,23 & ,24 &, 23 & 0,20 & 0,19 & 0,19 & 0,16 & 0,15 & 0,15 & 0,13 & & 0,08 & 0,0 \\
\hline$M_{4}$ & 00 & 00 & 00 & 0,17 &, 24 &, 30 & 0,34 & 0,34 & 0,35 & 0,38 & 0,39 & 0,40 & 0,40 & 40 & 0,41 & 0,4 \\
\hline$M_{5}$ & 00 & 00 &, 00 & 0,00 & ,05 &, 10 & 0,13 & 0,13 & 0,14 & 0,14 & 0,14 & 0,13 & 0,12 & 07 & 0,06 & 0,0 \\
\hline$M_{6}$ & 00 & 00 &, 00 & 0,00 &, 00 &, 02 &, 06 &, 06 & 0.06 & 0,05 & 0,04 & 0,05 & 05 & & 0,04 & 0,0 \\
\hline$M_{7}$ & 00 & 00 &, 00 & 0,00 & 0,00 & 0,00 & 0,02 & 0,02 & ה & 0,04 & 0,05 & & 06 & & ,06 &, 0 \\
\hline$M_{8}$ & 00 &, 00 & ,00 & 0,00 &, 00 & 0,00 & (20 & (200 & & $00 ?$ & 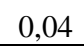 & & & & 0.07 & 0 \\
\hline$M_{9}$ & 00 &, 00 &, 00 & ת ת ת & ת & (200 & (20 & & & & & & & & & \\
\hline$M_{10}$ & 00 & 00 & 0,00 & 0,00 & , & , & , & 0,00 & & & 0,01 & & & & 0,10 & 0,0 \\
\hline$M_{11}$ & 00 & 0,00 & 0,00 & 0,00 &, 00 &, 00 & 0,00 & 0,00 & 0,00 & 0,00 & 0,00 & 0,03 & 0,04 & & 0,00 & 0,0 \\
\hline$M_{12}$ & 00 & 0,00 & 0,00 & 0,00 & ,00 &, 00 &, 00 & 0,00 & 0,00 & 0,00 & 0,00 & ,02 & ,03 & & 0,05 & 0,0 \\
\hline$M_{13}$ & 00 &, 00 &, 00 &, 00 & ,00 & ,00 &, 00 & ,00 & 0,00 & 0,00 & 0,00 & 0,00 &, 01 & 03 & 0,03 & 0,0 \\
\hline & 00 &, 00 &, 00 & $\underline{0,00}$ &, 00 &, 00 & 0,00 & 0,00 & 0.00 & 0,00 & 0,00 & 000 & $0 \Omega 0$ & & 0,06 & - \\
\hline$M_{15}$ & 00 &, 00 & $0 \Omega 0$ & ת & (0 & תم م & 0 & ת & 0 & $\cap \cap 0$ & ח 0 ח & & 000 & & 001 & 0 \\
\hline$M_{16}$ & 0,00 & 0,00 & 0,00 & 0,00 & 0,00 & 0,00 & 0,00 & 0,00 & 0,00 & 0,00 & 0,00 & 0,00 & $0 \Omega$ & & 0,00 & 0,0 \\
\hline$U(10)$ & 91 & 1,13 & 1,13 & 4,99 & 3,83 & 3,83 & 3,83 & 3,83 & 3,83 & 4,43 & 4,43 & 5,95 & 5,95 & & 6,83 & 0,8 \\
\hline$U(25)$ & 5,81 & 1,52 & 1,52 & 5,70 & 3,71 & 3,71 & 71 & 3,71 & 3,71 & 4,92 & 4,92 & 7,3 & 7,31 & & 7,67 & 7,6 \\
\hline 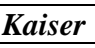 & 1 & 1 & 2 & & & & & & & & & & & & & 3 \\
\hline & 120 & 11 & 12 & & & & & & & & & & & & & \\
\hline & & 23 & & 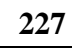 & & & & & 23 & & 23 & & 23 & & 235 & 23 \\
\hline SIC & 104 & 102 & 105 & 98 & 102 & 104 & 104 & 104 & 108 & 106 & 100 & 110 & 102 & 105 & 106 & 10 \\
\hline
\end{tabular}

Source : Bloomberg, données journalières du 02/09/2003 au 26/02/2016, calculs des auteurs. La première ligne du tableau donne les valeurs des différents paramètres $\delta$ pour les 16 indices $I d_{k}$ pour $k=1, \ldots 16$. Les 16 mesures de risque systémique sont représentées par $M_{1}$ à $M_{16}$ et correspondent dans l'ordre à : $M_{1}$ : Spillover Index, $M_{2}:$ HerfindahlHirschman Index, $M_{3}:$ VaR, $M_{4}$ : Absorption Ratio, $M_{5}:$ CoVaR, $M_{6}:$ MES, $M_{7}$ : Term Spread, $M_{8}$ : Default Yield Spread, $M_{9}$ : CES, $M_{10}$ : Volatility, $M_{11}$ : Dynamical Causality Index, $M_{12}$ : SRISK, $M_{13}$ : Turbulence Index, $M_{14}$ : $\triangle \mathrm{CoVaR}, M_{15}$ : TED Spread et $M_{16}$ : Amihud Illiquidity Measure. Les mesures actives dans la composante (par colonne) sont représentées en noir, tandis que celles qui sont inactives sont en gris. Les deux lignes suivantes du tableau donnent la valeur de la statistique du test de Hong et al. [2009] (notées $U(10)$ et $U(25)$ pour des valeurs du paramètre $d=10$ et $d=25$ ). L'indice optimal correspond à l'indice $I d_{14}$ dont les variables actives son en gras tout comme les statistiques pour des valeurs du paramètre de troncature $d=10$ et $d=25$. Le seuil pour la significativité au niveau de risque nominal de $5 \%$ est de 1,96 . Les quatre dernières lignes du tableau présentent le nombre de mesures respectant le critère de Kaiser et les valeurs des critères de parcimonie $A I C, B I C$ et SIC pour chacun des indices $I d_{k}$. Les valeurs en gras représentent les valeurs optimales au sens d'un des critères.

Les résultats du test de causalité à la Hong et al. [2009] pour les différents indices concurrents (notés de $I d_{1}$ à $I d_{16}$ ) sont résumés dans le tableau 1 , pour deux valeurs ${ }^{12} \mathrm{du}$ paramètre $d$, de $d=10$ et $d=25$. L'hypothèse nulle d'absence de causalité allant des variations mensuelles positives et extrêmes de chaque indice agrégé de risque systémique

\footnotetext{
${ }^{12}$ Nous avons également cherché à tester l’indice optimal pour un ensemble de valeurs du paramètre $d=10$ à $d=25$. Les résultats du test de causalité de Hong et al. [2009] pour ces valeurs concluent tous au choix de l'indice $I d_{14}$ comme indice optimal.
} 
vers les variations mensuelles négatives et extrêmes de la production industrielle, n'est rejetée que dans deux configurations au seuil nominal de 5\% (pour les modèles contenant 2 et 3 mesures).

De la lecture de ce tableau, il ressort que l'indice optimal issu de la méthodologie ACPP est l'indice agrégé $I d_{14}$. Nous notons que ce constat est valide quel que soit le paramètre $d$ retenu, avec des résultats relativement homogènes sur chaque ligne. Quelle que soit la valeur de $d$, cet indice apparaît en effet comme étant optimal au sens du test de Hong et al. [2009], avec une statistique de Hong et al. [2009], pour ce modèle, supérieure de plus de $30 \%$ à $50 \%$ à toutes les autres.

La lecture du tableau 1 indique que l'indice agrégé optimal selon le test de Hong et al. [2009] est principalement déterminé, dans l'ordre, par : le Spillover Index (avec un poids de 0,69), le Herfindahl-Hirschman Index (0,56), l’Absorption Ratio (0,40), la VaR $(0,10)$, la volatilité $(0,08)$, la CoVaR $(0,07)$ et le Default Yield Spread $(0,07)$. Un second ensemble de mesures vient s'ajouter dans sa composition, mais avec des poids relativement plus faibles comme dans le cas du Term Spread $(0,06)$, du Dynamical Causality Index $(0,06)$, la CES $(0,06)$, de la $\triangle \operatorname{CoVaR}(0,05)$, de la $\operatorname{SRISK}(0,04)$, de la MES $(0,04)$ et du Turbulence Index $(0,03)$. Le TED Spread et l'Amihud Illiquidity Measure sont deux mesures négligées.

Soulignons pour finir que les principales dimensions du risque systémique sont ainsi prises en compte dans notre indice final. Selon les critères d'information Akaike Information Criterion (AIC), Bayesian Information Criterion (BIC) et Schwartz Information Criterion (SIC), l'indice optimal ne serait composé que des quatre premières mesures citées plus haut. Selon le critère de Kaiser, seules les trois premières mesures doivent être prises en compte. Autrement dit, c’est la dimension du lien avec l'économie réelle qui nous contraint à être moins parcimonieux que ce qu'indiquent les critères d'information.

Le tableau 1 donne ainsi une information supplémentaire quant à l'application des différentes ACPP obtenues sous contrainte : elle concerne l'ordre d'importance ${ }^{13}$ des variables dans la composition de l'indice. Si une seule variable était à sélectionner, le choix se porterait sur le Spillover Index (seule variable non-nulle - en noir - dans la

${ }^{13}$ Il est important de noter que, du fait des multi-colinéarités présentes dans les mesures de risque systémique, chaque mesure est importante ici, indépendamment de leur poids ou de leur ordre d'entrée dans la composition de l'indice. 
première colonne, avec un coefficient de 1 dans le tableau 1). Viendront ensuite par ordre de pertinence : le Herfindahl-Hirschman Index, l'Absorption Ratio, la VaR, la volatilité, la CoVaR et le Default Yield Spread. Les deux dernières mesures à rentrer dans la composition de l'indice optimal seront le TED Spread et l'Amihud Illiquidity Measure.

L'indice optimal sera ainsi construit à partir de 14 mesures de risque systémique, dans le sens où celui-ci réalisera le meilleur compromis : la meilleure stabilité dans le temps et le pouvoir prédictif le plus élevé sur les contractions sévères de l'activité économique.

Le graphique 2 présente ainsi l'évolution temporelle de l’indice optimal constitué de 14 mesures de risque systémique sur les 16 au total, pour une valeur de $\delta=2,304$.

Maintenant que notre indice de risque systémique est construit, nous pouvons analyser plus avant dans la prochaine section le lien entre notre indice de risque systémique global et les valorisations boursières dans le cadre d'un modèle instantané usuel.

\section{Graphique 2 : Dynamique de l’indice agrégé optimal}

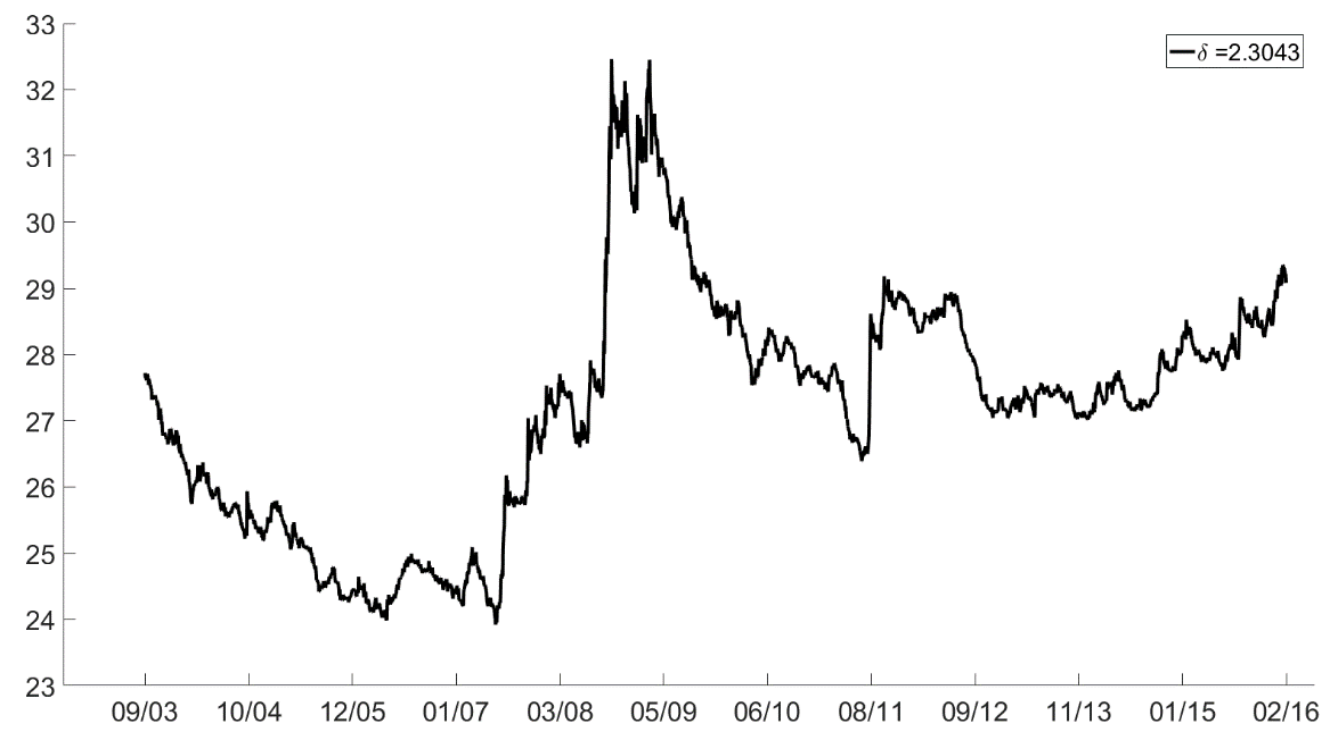

Source : Bloomberg, données journalières du 02/09/2003 au 26/02/2016 ; calculs des auteurs. L’indice obtenu par l'ACPP est composé de 14 mesures du risque systémique pour un paramètre $\delta=2,3043$.

Il s’agira de prendre en compte le risque systémique dans l'évaluation des actifs financiers traditionnelle et de voir si, d'une part, ce nouveau facteur de risque est significatif et, d’autre part, s’il permet de mieux expliquer la rentabilité des titres financiers sur le marché quand il est ajouté à un ensemble de facteurs de risque communs déjà bien illustrés dans la littérature. 


\section{Du MEDAF avec risque systémique}

Dans cette section, nous introduisons ainsi le Modèle d'Evaluation des Actifs Financiers avec risque Systémique (noté MEDAFS), pour inclure le risque systémique dans le MEDAF traditionnel.

\section{Du MEDAF traditionnel au MEDAF étendu au risque systémique}

Nous reprenons la théorie du Modèle d'Equilibre des Actifs Financiers (MEDAF) proposé par Sharpe [1964], Lintner [1965] et Mossin [1966], et son extension à trois facteurs de Fama et French [1993] prenant en compte certaines anomalies dans la valorisation des prix des actifs (Cf. Fama et French [1996]). Puis, nous introduisons ensuite un facteur lié au risque systémique dans le MEDAF étendu au modèle à trois facteurs de Fama-French [1993], au moyen d'un facteur additionnel correspondant à l’indice de risque systémique optimal construit dans la section précédente.

Nous obtenons ainsi un modèle à quatre facteurs - modèle (II) ci-dessous - en comparaison du modèle de Fama et French [1993] - modèle (I) ci-dessous, tels que :

$\left\{\begin{array}{l}r_{i, t}=\alpha_{i}^{(I)}+\beta_{i}^{(I)} r_{M, t}+\theta_{i}^{(I)} r_{S M B, t}+\gamma_{i}^{(I)} r_{H M L, t}+\varepsilon_{i, t}^{(I)} \\ r_{i, t}=\alpha_{i}^{(I I)}+\beta_{i}^{(I I)} r_{M, t}+\theta_{i}^{(I I)} r_{S M B, t}+\gamma_{i}^{(I I)} r_{H M L, t}+\varphi_{i}^{(I I)} r_{I S R M, t}+\varepsilon_{i, t}^{(I I)}\end{array}\right.$

où les facteurs au temps $t$, sont les rendements du marché $r_{M, t}$, les rendements du facteur $S M B$ notés $r_{S M B, t}$, ceux du facteur $H M L$ notés $r_{H M L, t}$ et où le facteur de risque systémique noté $r_{I S R M, t}$ représentant les rendements de l'indice optimal est le facteur supplémentaire dans la relation (II).

Une fois que nous avons écrit la relation de l'évaluation des actifs dans sa forme la plus simple, en insistant sur la relation linéaire entre le rendement et les primes, nous devons maintenant faire face à d'autres particularités des marchés financiers.

En particulier, nous devons d'abord prendre en compte dans la technique d'estimation les potentielles interrelations entre les rendements des actifs. Nous utilisons la méthode SURE de Zellner [1962] (pour Seemingly Unrelated Regressions Estimation) du modèle 
proposé (Cf. Kraus et Litzenberg, [1976] ; Barone-Adesi et al., [2003] ; Galagedera et Maharaj, [2008]) $)^{14}$.

Dans la suite sont reportés les tests empiriques du MEDAFS sur le marché américain des actions.

\section{Tests empiriques du MEDAFS sur le marché américain}

Nous comparons ci-après le modèle à trois facteurs de Fama et French [1993] (modèle I de l'équation (1)) au modèle étendu au risque systémique (modèle II de l'équation (1)), i.e. en rajoutant notre indice de risque systémique comme quatrième facteur, et nous évaluons les différences dans les valeurs des paramètres estimés, ainsi que les coefficients de détermination ajustés pour le MEDAF à trois facteurs de Fama et French [1993], avec ou sans prise en compte de l'indice de risque systémique comme facteur supplémentaire.

A la différence de la littérature sur les tests du MEDAF, qui consiste à tester l'équation de la prime de risque, nous cherchons à tester le MEDAFS, et en particulier la significativité du risque systémique. Pour ce faire, nous nous référons aux travaux de Black et al. [1972] et nous reprenons leur méthode de regroupement et de classement des titres en portefeuilles, de manière à limiter les risques d'estimation. Nous commençons tout d'abord par créer dix portefeuilles contenant des titres. Ces portefeuilles sont construits en deux étapes. Le calcul des $\beta$ de chaque titre par régression des moindres carrés est d'abord effectué, puis les titres sont regroupés dans 10 portefeuilles en fonction d'une règle de tri descendant sur les $\beta$. A la date $t$, le rendement de chaque portefeuille représente la moyenne des rendements de chacun des titres qui le compose.

Pour les analyses empiriques de cette section, nous utilisons la base de données journalière d'un panel de 60 institutions financières sur le marché américain ( $C f$. Brownlees et Engle [2017]). Cette base de données contient les prix de marché des différents titres et les différents ratios de la capitalisation boursière en fréquence journalière sur la période du 2 septembre 2003 au 26 février 2016, et sont extraits de la base Bloomberg.

\footnotetext{
${ }^{14}$ Par souci d'économie de place dans cet article, une annexe spécifique récapitulant les différents modèles et leur mode d'estimation est disponible sur demande auprès des auteurs.
} 
Dans un premier temps, nous avons estimé par Moindres Carrés Ordinaires (ligne par ligne) la relation du MEDAF du modèle (I) et du modèle (II) (Cf. Equation (1)). Dans ce cadre (simpliste), le facteur de risque systémique est du signe négatif attendu dans 9 cas sur 10 (seulement positive et proche de 0 pour le portefeuille 3), et il est significatif dans 7 cas sur 10, avec des statistiques brutes de Student ${ }^{15}$ supérieures à 3 dans 6 cas sur 10 . Par ailleurs, l'ajout du risque systémique comme facteur supplémentaire ne modifie que très légèrement l'estimation des autres paramètres de la relation canonique du MEDAF étendu au modèle à trois facteurs de Fama et French [1993]. Autrement dit et comme on pouvait l'anticiper, l'ajout du facteur de risque systémique se justifie a priori pleinement, et il ne bouleverse pas la relation traditionnelle du MEDAF.

Cependant, l'estimation de la relation canonique du MEDAF et de son extension au risque systémique doit prendre en compte les interconnections entre les titres, et les résultats de significativité doivent être corrigés des autocorrélations et de l'hétéroscédasticité des variables financières.

Nous fournissons dans le tableau 2 suivant, les estimations du modèle à trois facteurs de Fama et French [1993] et du modèle étendu au risque systémique (facteur supplémentaire) en utilisant cette fois la méthode SURE de Zellner [1962] en système, avec une estimation par les Moindres Carrés Généralisés (MCG), et en corrigeant les tstatistiques de Student par la méthode de Newey et West [1987] ${ }^{16}$.

Et les résultats sont alors surprenants car, 1) bien que tous les coefficients soient assez semblables à ceux de l'estimation simple précédente - i.e. similaires, et majoritairement de signe attendu, 2) le nombre de portefeuilles dans lesquels le facteur de risque systémique est significatif est alors divisé par deux, et 3) les seuls portefeuilles significativement exposés au risque systémique sont les portefeuilles à faible $\beta$. Ainsi, seuls 3 portefeuilles sont, au final, significativement sensibles au risque systémique. De plus, on notera aussi que, sur la période totale, le facteur $S M B$ n’est plus significatif pour l'ensemble des portefeuilles pour les deux modèles, alors que le nouveau facteur de risque

\footnotetext{
${ }^{15}$ Comme évoqué par Harvey et al. [2016], une valeur de t-statistique supérieure à 3 est exigée au seuil habituel de significativité (voir aussi Feng et al. [2017]).

${ }^{16}$ Nous avons aussi alternativement corrigé les statistiques de Student en utilisant la correction de White [1980] ; les statistiques obtenues sont très similaires à celles corrigées par Newey-West [1987] présentées dans chacune des régressions.
} 
systémique l'est pour quelques portefeuilles au moins. Enfin, tout naturellement, nous constatons que le facteur de marché reste, quant à lui, essentiel et très significatif.

Tableau 2 : Estimation du MEDAF à trois facteurs de Fama-French et du MEDAFS utilisant l'indice de risque systémique sur le marché américain (Méthode SURE avec MCG et correction des statistiques de Student pour l'autocorrélation et l'hétéroscédasticité)

Nous estimons les différents paramètres du MEDAF entre les rendements excédentaires de chaque portefeuille suivant différents facteurs, à 3 facteurs de Fama-French (modèle I), et à 3 facteurs de FamaFrench avec prise en compte du risque systémique (modèle II) dont la forme développée est la suivante :

\begin{tabular}{|c|c|c|c|c|c|c|c|c|c|c|c|}
\hline & \multicolumn{5}{|c|}{ Modèle I (modèle sans risque systémique) } & \multicolumn{6}{|c|}{ Modèle II (modèle avec risque systémique) } \\
\hline & $\alpha_{i}^{(I)}$ & $\beta_{i}^{(I)}$ & $\theta_{i}^{(I)}$ & $\gamma_{i}^{(I)}$ & $\overline{R^{2}}$ & $\alpha_{i}^{(I I)}$ & $\beta_{i}^{(I I)}$ & $\theta_{i}^{(I I)}$ & $\gamma_{i}^{(I I)}$ & $\varphi_{i}^{(I I)}$ & $\overline{R^{2}}$ \\
\hline Groupe 1 & 5,88** & $\overline{1} 1,12^{* * *}$ & $-0,06$ & $-1,16 * * *$ & $70,12 \%$ & $5,83 * *$ & 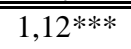 & 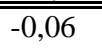 & $-1,15^{* * *}$ & "-0,12 & $70,12 \%$ \\
\hline Groupe 2 & $5,95 * *$ & $1,01 * * *$ & 0,00 & $-1,07 * * *$ & $68,61 \%$ & $5,77 * *$ & $1,01 * * *$ & 0,00 & $-1,06 * * *$ & $-0,18$ & $68,67 \%$ \\
\hline Groupe 3 & 3,80 & $0,95 * * *$ & $-0,04$ & $-1,05 * * *$ & $72,10 \%$ & 3,93 & $0,95 * * *$ & $-0,04$ & $-1,05 * * *$ & 0,12 & $72,10 \%$ \\
\hline Groupe 4 & 0,96 & $0,95 * * *$ & $-0,06$ & $-0,95 * * *$ & $71,48 \%$ & 0,82 & $0,94 * * *$ & $-0,07$ & $-0,94 * * *$ & $-0,30$ & $71,71 \%$ \\
\hline Groupe 5 & $6,53 * * *$ & $0,89 * * *$ & 0,03 & $-0,72 * * *$ & $60,40 \%$ & $6,42 * * *$ & $0,88 * * *$ & 0,03 & $-0,71 * * *$ & $-0,05$ & $60,41 \%$ \\
\hline Groupe 6 & 3,71 & $0,87 * * *$ & $-0,10$ & $-0,87 * * *$ & $66,50 \%$ & 3,62 & $0,86 * * *$ & $-0,11$ & $-0,86 * * *$ & $-0,20$ & $66,61 \%$ \\
\hline Groupe 7 & $3,76^{* *}$ & $0,80 * * *$ & 0,11 & $-0,42 * * *$ & $56,88 \%$ & $3,69 * *$ & $0,78 * * *$ & 0,10 & $-0,41 * * *$ & $-0,32 * * *$ & $57,12 \%$ \\
\hline Groupe 8 & 3,87 & $0,78 * * *$ & 0,01 & $-0,76 * * *$ & $56,58 \%$ & 3,30 & $0,76 * * *$ & $-0,01$ & $-0,73 * * *$ & $-0,50 * * *$ & $57,07 \%$ \\
\hline Groupe 9 & $-1,08$ & $0,73 * * *$ & 0,02 & $-0,32 * * *$ & $51,42 \%$ & $-1,51$ & $0,71 * * *$ & 0,00 & $-0,29 * * *$ & $-0,53 * * *$ & $52,54 \%$ \\
\hline Groupe 10 & 3,40 & $0,62 * * *$ & 0,05 & $-0,10$ & $32,35 \%$ & 3,20 & $0,61^{* * *}$ & 0,04 & $-0,09$ & $-0,26$ & $32,58 \%$ \\
\hline
\end{tabular}

Source : Bloomberg, la base de données comprend un ensemble complet de prix des titres de notre base de données américaine comprenant 60 institutions financières en données quotidiennes du 2 septembre 2003 au 26 février 2016. Calculs des auteurs. Le tableau donne les valeurs des différents paramètres du MEDAF à trois facteurs de Fama et French [1993] sans risque systémique (modèle I) et avec prise en compte du risque systémique (modèle II). Les valeurs du paramètre $\alpha$ sont exprimées en $10^{-4}$. L'estimation des paramètres se fait par la méthode SURE de Zellner [1962], en système avec MCG, et correction des statistiques de Student pour l'hétéroscédasticité par la méthode de NeweyWest [1987]. Les seuils usuels de significativité sont fixés respectivement à $10 \%, 5 \%$ et $1 \%$ pour *, ** et ***.

Le graphique 3 représente l'évolution de la relation empirique entre les rendements moyens et le niveau de la prime de risque liée au facteur de marché, le paramètre $\beta$, dans différents environnements de risque systémique. Il est à noter que la relation s’inverse et qu'elle devient négative quand le risque systémique est fort. Ainsi, dans un environnement de risque systémique faible - voir le cadrant supérieur gauche, un portefeuille avec un $\beta$ élevé aura des rendements attendus plus élevés qu'un portefeuille ayant un $\beta$ faible. Dans un environnement de risque systémique élevé par contre - voir le cadrant inférieur droit, un portefeuille avec un $\beta$ élevé aura des rendements attendus plus faibles, voir négatifs, que ceux des portefeuilles ayant un $\beta$ faible.

Ces derniers éléments d'analyse montrent que les relations entre le risque systématique et le risque systémique, et de manière plus générale les relations entre les 
différents facteurs, sont plus complexes qu’anticipées, ce qui nous invite à tenter d’isoler l’impact du risque systémique.

\section{Graphique 3 : Evolution de la relation empirique entre les rendements moyens et le facteur de risque de marché dans divers environnements de risque systémique}
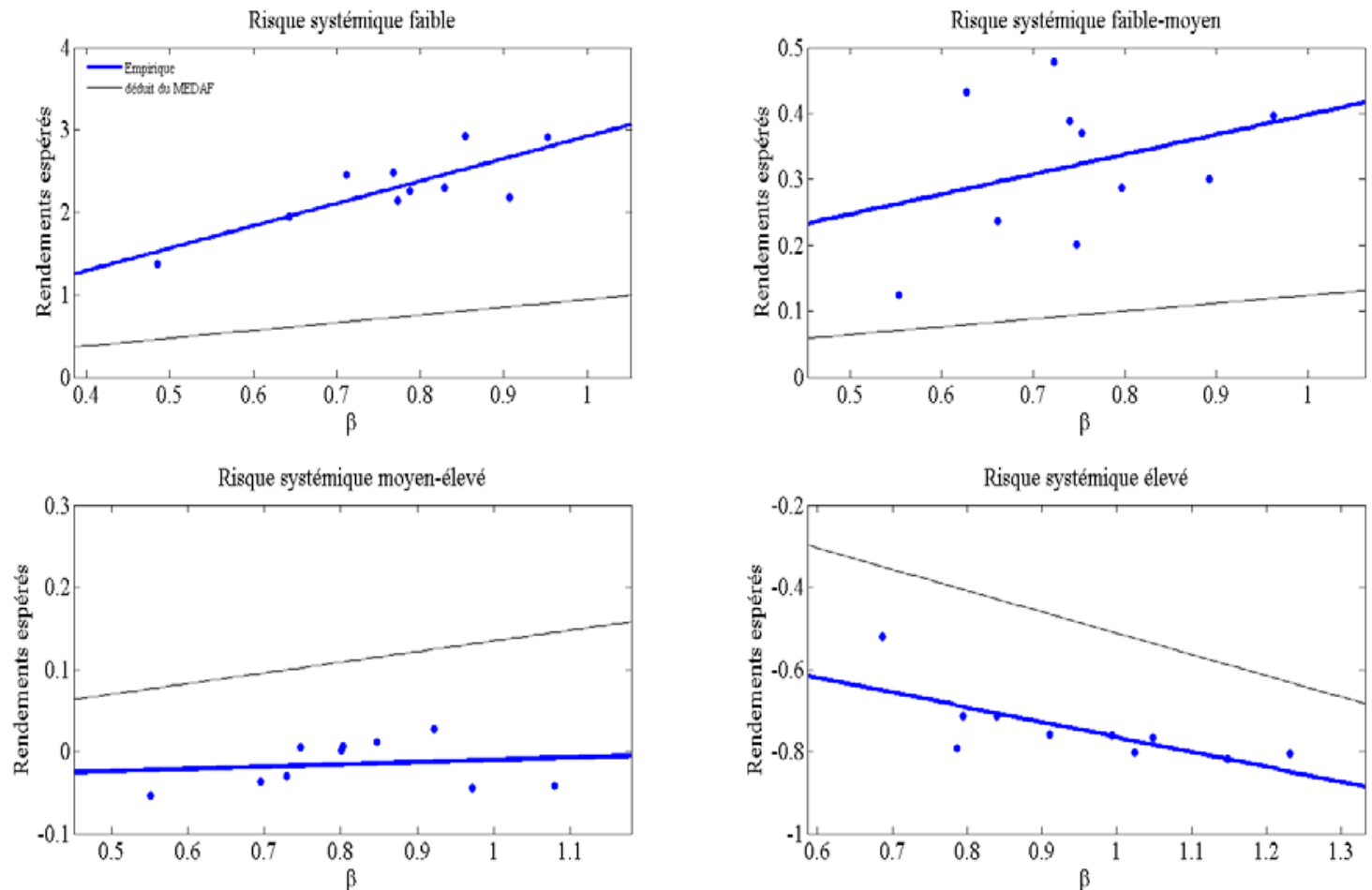

Source : Bloomberg, la base de données comprend un ensemble complet de prix des titres de notre base de données américaine comprenant 60 institutions financières en données quotidiennes du 2 septembre 2003 au 26 février 2016. Calculs des auteurs. Nous créons tout d'abord dix portefeuilles à partir des classements sur les bêtas estimés par rapport au marché. Le graphique représente les relations rendement-facteur-risque. La période risque systémique faible correspond à un facteur de risque systémique $r_{I S R M, t} \in[-0,0227,-0,0012$ [ , la période risque systémique faiblemoyen correspond à $r_{I S R M, t} \in\left[-0,0012,-0,00015\right.$ [, la période risque systémique moyen-élevé à $r_{I S R M, t} \in$ $\left[-0,00015,0,00089\right.$ [ et la période risque systémique élevé correspond à $r_{I S R M, t} \in[0,00089,0,0413[$. L'axe des abscisses représente le niveau des bêtas des différents portefeuilles et l'axe des ordonnées, les rendements espérés annualisés. La ligne fine est la relation prédite par le MEDAF et la ligne en gras est calculée par régression des rendements moyens sur les différents bêtas.

Dans la suite ${ }^{17}$, le tableau 3 compare plus avant le MEDAFS (modèle II du tableau 2) à un MEDAFS, dans lequel cette fois les facteurs ont été préalablement orthogonalisés

\footnotetext{
${ }^{17}$ Nos premiers résultats globaux, significatifs mais sensiblement mitigés quand les facteurs ne sont pas préalablement orthogonalisés, nous ont amené tout naturellement à conduire quelques tests supplémentaires de robustesse. Au final, nous avons exprimé l'extension du MEDAF avec prise en compte du risque systémique au travers de plusieurs modèles avec décontamination des facteurs, et avec un modèle multifactoriel de type Modèle d'Evaluation par Arbitrage (voir Ross [1976] et Chen et al. [1986]) sur les titres et sur les portefeuilles. Les résultats obtenus confortent tous l’idée de la nécessaire prise en en compte du risque systémique dans le MEDAF traditionnel puisque le facteur de risque systémique est significatif pour une grande majorité des portefeuilles, du signe (négatif) attendu, avec des statistiques de Student très élevées.
} 
(modèle III du tableau 3). Orthogonaliser les facteurs a ici pour but d'isoler au mieux l'effet final du risque systémique sur les rentabilités boursières, en atténuant (partiellement au moins) le problème de colinéarité et en ne traitant alors que des variables orthogonales. Le tableau 3 renforce l’interprétation de nos précédents résultats, puisque, comme attendu, l'effet du risque systémique, maintenant isolé, est très significatif pour l'ensemble des portefeuilles (avec des statistiques de Student largement supérieures à 3).

\section{Tableau 3 : Estimation du MEDAF et du MEDAFS avec orthogonalisation des trois facteurs du modèle de Fama-French, en utilisant l'indice de risque systémique sur le marché américain (Méthode SURE avec MCG et correction des statistiques de Student pour l'autocorrélation et l'hétéroscédasticité).}

Nous estimons les différents paramètres du MEDAF entre les rendements excédentaires de chaque portefeuille suivant différents facteurs, à 3 facteurs de Fama-French (modèle I), et à 3 facteurs de FamaFrench avec prise en compte du risque systémique et orthogonalisation préalable des facteurs (modèle III), dont la forme développée est ici la suivante :

$$
r_{i, t}=\alpha_{i}^{(I I I)}+\beta_{i}^{(I I I)} \hat{\varepsilon}_{M, t}+\theta_{i}^{(I I I)} \hat{\varepsilon}_{S M B, t}^{\circ 0}+\gamma_{i}^{(I I I)} \hat{\varepsilon}_{H M L, t}^{000}+\varphi_{i}^{(I I I)} r_{I S R M, t}+\varepsilon_{i, t}^{(I I I)}
$$

avec :

$$
\left\{\begin{array}{l}
\hat{\varepsilon}_{M, t}=r_{M, t}-\hat{b}_{M} r_{I S R M, t} \\
\hat{\varepsilon}_{S M B, t}^{\circ 0}=r_{S M B, t}-\left(\hat{b}_{S M B}^{\circ} r_{M, t}+\hat{b}_{S M B}^{\circ \circ} r_{I S R M, t}\right) \\
\hat{\varepsilon}_{H M L, t}^{\circ 00}=r_{H M L, t}-\left(\hat{b}_{H M L}^{\circ} r_{M, t}+\hat{b}_{H M L}^{\circ \circ} r_{S M B, t}+\hat{b}_{H M L}^{\circ 00} r_{I S R M, t}\right) .
\end{array}\right.
$$

\begin{tabular}{|c|c|c|c|c|c|c|c|c|c|c|c|c|}
\hline & \multicolumn{6}{|c|}{ Modèle II (facteurs bruts) } & \multicolumn{6}{|c|}{ Modèle III (facteurs orthogonalisés) } \\
\hline & $\alpha_{i}^{(I I)}$ & $\beta_{i}^{(I I)}$ & $\theta_{i}^{(I I)}$ & $\gamma_{i}^{(I I)}$ & $\varphi_{i}^{(I I)}$ & $\overline{R^{2}}$ & $\alpha_{i}^{(I I I)}$ & $\beta_{i}^{(I I I)}$ & $\theta_{i}^{(I I I)}$ & $\gamma_{i}^{(I I I)}$ & $\varphi_{i}^{(I I I)}$ & $\overline{R^{2}}$ \\
\hline & 5,83** & $1,12^{* * *}$ & $\begin{array}{l}-0,06 \\
\end{array}$ & $-1,15^{* * *}$ & $\overline{0,12}$ & ,12\% & $5,53 * *$ & $1,1,49 * * *$ & $0,28 * * *$ & ,15*** & ,31*** & 0,12 \\
\hline & $77 * *$ & $1 * * *$ & 0,00 & $06 * * *$ & 18 & & 5,49 & $1,35^{* * *}$ & & $-1,06 * * *$ &, $22 * * *$ & \\
\hline & 3,93 & $0,95 * * *$ & $-0,04$ & $-1,05 * * *$ & 0,12 & $\%$ & 3,67 & $1,29 * * *$ & $0,27 * * *$ & $-1,05 * * *$ & $-1,83 * * *$ & 72,10\% \\
\hline & 82 & $4 * * *$ & $-0,07$ &, $94 * * *$ & & & 0,58 & $1,24 * * *$ & 0,20 & $-0,94 * *$ & $-2,09$ & 1,11 \\
\hline & $6,42 * * *$ & $0,88 * * *$ & 0,03 &, $71 * * *$ & $-0,0$ & & $6,19 * *$ & $1,11^{* * *}$ & $* * *$ & $-0,71 * * *$ & $1,66^{* * *}$ & $1 \%$ \\
\hline & 3,62 & $0,86 * * *$ & $-0,11$ & $-0,86 * * *$ & $-0,20$ & & 3,40 & $1,14^{* * *}$ & & $-0,86 * * *$ & $-1,81 * * *$ & $1 \%$ \\
\hline & $3,69 * *$ & $0,78 * * *$ & 0,10 & $-0,41 * * *$ & $-0,32 * *$ & & $3,49 * *$ & $0,91 * * *$ & $0,21 * * *$ & $-0,41 * * *$ & $-1,57 * * *$ & $57,12 \%$ \\
\hline & 3,30 & $0,76 * * *$ & $-0,01$ & $-0,73 * * *$ & & & 3,09 & $1,00^{* * *}$ & $0,21 * * *$ & $-0,73 * * *$ & $-1,97 * * *$ & 7,079 \\
\hline & $-1,51$ & $0,71 * * *$ & 0,00 & $-0,29 * * *$ & $-0,53 * * *$ & & $-1,67$ & $0,80 * * *$ & 0,08 & $-0,29 * * *$ & $-1,51 * * *$ & $2,54 \%$ \\
\hline sroupe 10 & 3,20 & $0,61 * * *$ & 0,04 & $-0,09$ & $-0,26$ & $32,58 \%$ & 3,06 & $0,64 * * *$ & 0,06 & $-0,09$ & $-0,98 * * *$ & $32,58 \%$ \\
\hline
\end{tabular}

Source : Bloomberg, la base de données comprend un ensemble complet de prix des titres de notre base de données américaine comprenant 60 institutions financières en données quotidiennes du 2 septembre 2003 au 26 février 2016. Calculs des auteurs. Le tableau donne les valeurs des différents paramètres du MEDAF à trois facteurs de Fama et French [1993] avec risque systémique (modèle II) en facteurs bruts et avec facteurs orthogonalisés (modèle III). Les valeurs du paramètre $\alpha$ sont exprimées en $10^{-4}$. L'estimation des paramètres se fait par la méthode SURE de Zellner [1962] en système avec MCG, et correction des statistiques de Student pour l'hétéroscédasticité par la méthode de Newey-West [1987]. Les seuils usuels de significativité sont fixés respectivement à $10 \%, 5 \%$ et $1 \%$ pour $*$, ** et $* * *$.

Ainsi, il ressort au final de notre analyse que le risque systémique apparaît 1) comme significatif dans l'estimation de la relation canonique du MEDAF étendue à ce nouveau facteur et que 2) les différents facteurs traditionnels semblent interférer avec ce facteur. A l'instar de ce qu'écrivent Billio et al. [2017], il existerait ainsi plusieurs composantes 
dans les rentabilités boursières : une composante systématique et systémique (liée aux marchés en période de crise), une composante systématique et non-systémique (liée aux marchés dans des conditions «normales »), une composante spécifique et systémique (liée aux titres individuels et à leurs potentielles sensibilités propres au risque systémique), une composante spécifique et non-systémique (liée à des caractéristiques idiosyncratiques des différents titres).

Plus précisément, Billio et al. [2017] envisagent une relation telle qu’en exprimant le modèle (I) sous forme matricielle et en prenant en compte les interconnexions entre les actifs $^{18}$, qui pondéreraient en quelque sorte l'importance des différentes rentabilités dans les relations canoniques des rentabilités boursières ; les auteurs rendent ainsi explicite une décomposition du risque total en quatre sources de risque distinctes, telles que : les risques systématiques liés au marché, les risques spécifiques liés aux firmes, l’impact du risque systémique sur le marché, et l'impact du risque systémique sur les différents risques idiosyncratiques. En additionnant les impacts du risque systémique sur les différents risques systématiques et idiosyncratiques, ils obtiennent alors la contribution totale du réseau (et donc du risque systémique) au risque total, qui n’est autre que la somme de quatre composantes de risque susmentionnées. Dans le cadre de notre analyse, dans laquelle le nombre de connections inter-institutions intervient dans la construction de l'indice (au travers de la prise en compte du DCI de Billio et al. [2012]) le risque systémique est, ici, ajouté comme nouveau facteur explicatif de la rentabilité des titres et il prend en compte non seulement l'interconnexion mais aussi d'autres aspects de ce risque. Ainsi, à l'instar de Billio et al. [2017], nous proposons de prendre en compte le risque systémique dans l'évaluation des prix des actifs. Toutefois, nous choisissons l'ajout d'un facteur supplémentaire et proposons d'en obtenir l'effet « net » sur la rentabilité des titres a contrario de Billio et al. [2017] qui optent pour une recherche des effets des différents facteurs « augmentés » du risque systémique.

Une fois la validation du MEDAFS désormais vérifiée, nous proposons dans la suite une application originale de ce modèle, à travers l'identification et le classement des Institutions Financières d'Importance Systémique (IFIS).

\footnotetext{
${ }^{18}$ Nous renvoyons le lecteur intéressé à une annexe disponible sur demande auprès des auteurs, qui contient une description sommaire de l'écriture du modèle sous-jacent.
} 


\section{De la détermination des institutions financières d'importance systémique}

La crise mondiale débutée en 2007 a mis en exergue la nécessité de renouveler les approches de régulation, jusque-là essentiellement micro-prudentielles, en lui ajoutant une dimension macro-prudentielle. L'objectif est ici de corriger certaines défaillances des marchés financiers, dont le risque global n’est pas seulement l'agrégation des risques individuels relatifs aux différentes institutions qui composent le système financier. Le risque ultime qui pèse sur le système financier est ainsi systémique, car il conduirait en cas de réalisation extrême à l'effondrement du système dans son ensemble, du fait des interdépendances et interactions complexes qui unissent ses composantes.

Ainsi, la politique macro-prudentielle trouve sa pleine justification dans le constat que la régulation micro-prudentielle (telle que présentée dans les accords de Bâle par exemple), bien que nécessaire, n'est pas suffisante pour garantir la stabilité d’ensemble du système financier. En effet, certains aspects de la régulation micro-prudentielle, qui visent à surveiller et à protéger les institutions individuelles, peuvent paradoxalement déstabiliser le système financier dans sa totalité du fait de leur caractère pro-cyclique (Daníelsson [2002] ; Hanson et al. [2011]). A titre d'exemple, des ratios de fonds propres minimums fixés indépendamment du déroulement du cycle d’activité peuvent être une source de risque systémique. Le respect, coûte que coûte, de ces ratios sont en effet susceptibles de pousser les institutions à se désendetter et à vendre des actifs risqués dans les mauvais états de la nature, dans des conditions souvent peu avantageuses pour elles dans le cadre de «vente forcées » (Fire Sales en anglais - Cf., e.g., Cont et Wagalath [2013]), tendant à faire chuter les prix et à aggraver ainsi la crise dans une sorte de cercle vicieux. Dans certaines conditions, ces actions aboutiraient non pas à augmenter les fonds propres des institutions pour satisfaire aux ratios prudentiels, mais au contraire à fragiliser encore plus ces mêmes institutions. Ainsi, la dimension endogène des risques pesant sur le système financier résulte du comportement collectif des agents et des institutions financières, et le risque systémique n'est pas seulement la résultante de la somme des risques individuels mesurés en dehors de toute autre considération.

Parmi les récentes initiatives en matière de régulation macro-prudentielle, un effort particulier a été dernièrement consacré à la détection des IFIS (e.g. Benoit et al. [2017b]). 
Pendant longtemps, identifier les institutions d’importance systémique revenait simplement à apprécier la taille du bilan des banques dans une logique de type «tropgrosse-pour-faire-faillite » (en anglais «too-big-to-fail »). Il n'était pas en effet sans conséquence évidente de laisser une grande institution faire faillite, ce qui interdisait de fait sa faillite. Mais la dernière crise de 2007-2008 a mis en évidence d'autres sources de fragilité du système : elle a montré notamment que certains établissements très (trop) interconnectés faisaient peser aussi un risque sur le système financier dans son ensemble.

Une limite supplémentaire concerne l'applicabilité de ces mesures. Le risque systémique ne se limite pas aux seules banques, mais concerne l'ensemble des institutions financières intervenant sur le marché (les compagnies d'assurance, les fournisseurs d'infrastructures de marché, les gérants d’OPCVM, etc.). Or la plupart des mesures ont été conçues pour des institutions financières cotées (institutions financières et compagnies d'assurance). Des exemples montrent que certaines institutions, non cotées, font courir autant, voir plus de risque au système que d'autres qui sont elles cotées. A l'inverse, la mesure du risque systémique faite à partir d'informations privées est peut-être plus complète, mais la difficulté de consolider un certain nombre de positions la rend particulièrement sensible au risque de modèle. Par ailleurs, de grandes entreprises, nonfinancières, seraient signalées comme systémiques si les critères usités leur étaient appliqués. Enfin, certaines particularités organisationnelles d'institutions financières nationales (en France notamment) ont pour conséquences, peu ou prou, de mettre en avant un risque systémique important.

Nous devons aussi nous interroger sur le comportement stratégique optimal du régulateur, au sens de la théorie des jeux, qui se retrouve en quasi conflit d’objectifs lorsqu’il doit évaluer le risque systémique et la fragilité des institutions financières. Celuici est pris entre la tentation d’être sévère pour être crédible et celle de ne pas l'être pour éviter de créer lui-même un scenario de stress. De même, certains pourraient s'interroger, quelle que soit l'approche retenue pour identifier une institution systémique, sur l'utilité de publier officiellement une liste d'institutions systémiques. Cette publication rend plus explicite un sauvetage en cas de défaillance et cela rassure certes les marchés ; elle exacerbe toutefois le problème potentiel d’aléa moral (Bongini et al. [2015]). Certains auteurs (e.g. Moenninghoff et al. [2016]) montrent ainsi que la désignation officielle des banques comme «systémiquement importantes » a été suivie par une augmentation de 
leur valeur de marché. Cet avantage en termes de coût de financement est évalué par certains, même si l'exercice est difficile et périlleux, à quelques dizaines de points de base avant la crise, avec une augmentation nette et significative de cette prime après la crise (e.g. Ueda et Weder di Mauro, [2013]). Cette sorte de «garantie implicite » se traduit ainsi par une subvention (cachée et pour d'aucun indue) aux institutions systémiques en particulier, et au système financier dans son ensemble en général. Pire encore, les produits financiers conçus et les opérations initiées par les entités désignées comme systémiques voient leur risque perçu diminuer, ce qui réduit, en apparence du moins, le risque global du système et renforce la position de marché de l'institution. Il existerait ainsi une prime à être systémique.

Néanmoins, dans un cadre où certains effets pervers sont sous contrôle (limitation de l'incitation à prendre des risques) et l'existence de lacunes relatives bien identifiées (absence de cotation de certains acteurs, régulation du secteur de l'assurance, effet potentiels dans d'autres branches d'activité que celle strictement financière, existence de shadow banking, qualité de l'information collectée...), les avantages d'une mise en application transparente et systématique, sur des critères admis, d'une régulation pertinente, nous semblent d'une plus grande importance en comparaison d'éventuelles effets néfastes de la mise en place de cette surveillance (Le Pan [2016]). C'est aussi dans ce contexte que nous proposons dans la troisième partie, une méthodologie claire, justifiée, transparente et évolutive, visant à détecter les institutions financières susceptibles de fragiliser le système.

Ainsi, l'objectif final est désormais de détecter les IFIS sur un ensemble de critères (e.g., Das [2016]), en vue de leur appliquer des mesures spécifiques de supervision qui tendent à réduire les risques engendrés par ces institutions, et à limiter l'aléa moral qui résulte de la potentielle garantie publique implicite que reçoivent les institutions perçues comme « trop grosses, trop spéculatives, trop complexes et/ou trop interconnectées ${ }^{19}$.

Dans la prochaine sous-section, nous proposons dans ce cadre une application originale du MEDAFS, qui consiste à mesurer l'importance d'une institution financière au travers de sa sensibilité au facteur de risque systémique.

\footnotetext{
${ }^{19}$ A titre d'information, voir les propositions résultant du « CFP SIFI Challenge 2016 » lancé par le MIT Center for Finance and Policy (MIT CFP), ayant pour objectif d'améliorer la définition et l'identification des IFIS : http://cfpweb.mit.edu/BackgroundInformation/.
} 


\section{Qu'est-ce qu'une institution financière d'importance systémique ?}

L’importance systémique d'une institution devrait être expliquée à travers le risque que la possible faillite de celle-ci conduise à l'effondrement du système. Les grandes banques, les compagnies d'assurance, les systèmes de paiement au niveau des contreparties, les sociétés financières et les fonds d'investissement sont des exemples naturels de candidats potentiels pouvant être considérés comme des Institutions Financières d'Importance Systémique (IFIS). Afin de pouvoir déterminer quelles institutions peuvent être actuellement considérées comme des IFIS, il est toutefois nécessaire de définir quelles sont les caractéristiques principales du risque systémique et comment les mesurer.

Comme évoqué précédemment, le principe du «too-big-to-fail » était dominant jusqu’à récemment. En effet, une institution financière était considérée comme systémique par le simple fait de constater sa taille importante. Cependant, d'autres caractéristiques comme le «too-interconnected-to-fail », l'effet de levier, l'exposition au marché, les états conditionnels du marché et la complexité d'une institution doivent aussi être prises en compte, en plus de sa taille, pour juger si celle-ci est ou non une IFIS. Ces différents aspects sont cruciaux et c'est la raison pour laquelle plusieurs mesures du risque systémique ont récemment émergées de la littérature académique comme évoqué dans la première section de cet article. Jusqu’à récemment, ces mesures ont été implémentées séparément, en dépit du fait que le risque systémique est clairement un phénomène multidimensionnel.

Plus généralement, on peut distinguer deux approches traditionnelles pour déterminer si une institution financière est une IFIS ou non : les méthodes relatives à la construction d’indicateurs et les méthodes relatives à la modélisation du risque systémique.

La première approche fait état de facteurs liés à la contribution d'une institution au risque systémique, et repose sur un score ad hoc qui est utilisé pour déterminer si cette institution est une IFIS. Par exemple, les mesures basées sur la taille d'une institution et sa résilience aux échéances de dettes de courte maturité, peuvent être pondérées et combinées afin d’obtenir un score du risque systémique lié à cette institution. Si ce score est au-dessus d'un certain seuil, alors cette institution sera identifiée comme étant une 
IFIS. Une question importante est de se demander comment de tels critères devraient être choisis, mesurés, et pondérés pour différents types d’institutions financières.

La seconde approche tient à la modélisation des différentes interconnexions entre les institutions. Les modèles sont calibrés en utilisant des données et des scénarios de crise simulés afin d'estimer l'ampleur des pertes potentielles et les effets de contagion entre les institutions. Ces estimations sont ainsi utilisées pour déterminer la contribution d'une institution aux pertes liées à un événement systémique et son statut en tant qu’IFIS.

Actuellement, les régulateurs emploient typiquement les approches liées à la construction d'indicateurs pour identifier les IFIS. Ces modèles sont considérés comme étant les plus simples à administrer. Bien qu’une approche de modélisation complète des interconnexions entre les institutions soit considérée comme n’étant pas encore réalisable à ce stade (car trop complexe à mettre en œuvre), la combinaison des techniques ou l'incorporation de résultats de modèles comme facteurs dans un modèle d'indicateurs devraient aider à rapprocher ces deux approches traditionnelles.

Nous proposons ainsi dans la section suivante une autre approche, complémentaire, qui vise à mesurer la sensibilité effective de telle ou telle institution au risque systémique global, tel que mesurée par notre indice (ISRM pour Index of Systemic Risk Measures), comme définit dans notre première section, dans le cadre du MEDAFS présenté dans la deuxième section de cet article. Cette sensibilité au risque systémique nous servira de proxy de l'importance systémique de cette institution et il permettra d'établir un classement des IFIS comme expliqué dans les sections suivantes.

\section{Méthodologie d'identification des IFIS selon le MEDAFS}

Notre méthodologie comporte ainsi 5 étapes. Tout d'abord (Cf. la première section de l'article), un panel de mesures de risque systémique est calculé, chacune mettant l'accent sur tel ou tel aspect du risque systémique qui caractérisent les institutions financières. Deuxièmement, nous regroupons les informations extraites à partir de ce panel défini lors de la première étape, en utilisant la technique de réduction de dimension, à savoir l'ACPP. Une fois que nous avons identifié les principales composantes du modèle, nous procédons, dans la troisième étape, à la construction d'un indice agrégé des mesures de risque systémique (dénommé ISRM) en utilisant une somme pondérée 
particulière de ces composantes, reliant le choix final de l'ACPP déterminé par le plus fort lien possible des variations extrêmes de l'indice et du PIB futur. Plus précisément, nous évaluons lequel des indices (parmi les différents candidats potentiels) peut être considéré comme le meilleur indicateur avancé des chocs sur l'activité économique résultant d'un choc systémique. Nous utilisons pour cela le test de causalité extrême de Hong et al. [2009]. Quatrièmement, nous ajoutons l'ISRM optimal dans le modèle à trois facteurs de Fama-French [1993] et nous obtenons une nouvelle prime de risque : la prime de risque systémique. C'est alors, en cinquième étape, que nous obtenons la sensibilité (et la significativité) des institutions financières à cette prime de risque systémique en procédant effectivement aux estimations des différentes sensibilités aux facteurs des institutions financières. Ce qui nous donne un critère de classement objectif de l'importance systémique des institutions financières. Ainsi, plus la sensibilité au risque systémique est élevée pour un seuil de significativité donné, et plus l’institution financière aura un caractère systémique d'importance.

Bien sûr, des distinctions entre les institutions d'importance systémique au niveau national par rapport à celles d'importance systémique au niveau global devraient être prévues et peuvent être obtenues à la fois 1) en sélectionnant les institutions (et l'indice de marché) à partir d'un marché mondial ou national ou d'un secteur économique spécifique, et 2) en construisant un indice basé sur des mesures de risque systémique pertinentes pour le marché étudié.

On notera également ici que la désignation des IFIS selon cette méthodologie n'est pas figée dans le temps. Ainsi, une institution financière ayant une sensibilité qui augmente (ou qui baisse) sensiblement, pourrait changer : devenir IFIS (et être mise sous surveillance) si elle ne l'était pas avant, ou ne plus être IFIS si elle l'était avant. Au gré des conditions de marché (et du niveau global du risque systémique), et suite aux actions entreprises qui conduisent à réduire ou augmenter les risques, la désignation de IFIS pourrait être rendu dynamique, claire et explicite. Notons aussi que toutes les données utilisées sont publiques et que la méthodologie est facile à implémenter. Le modèle et la méthodologie pourraient ainsi être exécutés chaque trimestre (chaque année), et le classement adapté aux nouvelles conditions du marché et aux situations précises des institutions financières. 
Classer les potentielles IFIS par leurs sensibilités au risque systémique doit, évidemment, prendre en compte le niveau de significativité des sensibilités. Mais, la sévérité de la significativité retenue $(0,1 \% ; 1 \% ; 5 \% ; 10 \%, \ldots)$ doit aussi être adaptée aux conditions du marché et aux ressources des organismes de réglementation. Dans notre proposition, un certain pourcentage des principales IFIS classées en fonction de l'importance de leurs sensibilités au risque systémique devra être choisi (60\% dans notre illustration ${ }^{20}$ ), de manière à ne se focaliser que sur les plus systémiques. Dans ce cas, les plus importantes IFIS détectées par l'Office of Financial Research $(O F R)^{21}$ sont présentes dans l'échantillon des IFIS signalées par notre méthodologie.

La principale idée derrière cette méthodologie est que si les régulateurs peuvent imposer une réduction des mesures de risque systémique à chaque institution détectée comme des IFIS et suivie dans un processus de contrôle, alors l'indice de risque systémique, et donc le risque systémique global, diminueront également. En agissant sur les principaux contributeurs de l'indice, les autorités pourraient ainsi réduire le risque global, et donc, en retour, les risques et sensibilités des différents intervenants, jusqu'à obtenir un risque global jugé raisonnable et supportable par le système dans son ensemble. Ceci en concentrant les forces du régulateur, forcément limitées, sur les principales sources de risque systémique.

Le nombre d'IFIS selon nous, devrait être 1) suffisamment grand pour éviter de négliger un acteur important ; 2) assez grand pour pouvoir contrôler le risque global en imposant des restrictions marginales sur les différents aspects du risque systémique mesurés pour un sous-ensemble d'institutions (les IFIS); 3) évolutif de manière rationnelle et transparente pour limiter l'aléa moral ; et 4) sans être trop grand toutefois pour utiliser de manière efficace les ressources des régulateurs.

\footnotetext{
${ }^{20}$ Les scores annuels de risque systémique pour les principales banques à travers le monde sont tous basés sur les mêmes indicateurs. Aux États-Unis, chaque institution financière ayant plus de 50 milliards de dollars d'actifs doit divulguer annuellement ses indicateurs de risque systémique au régulateur ; elles sont disponibles sur le National Information Center :

https://www.ffiec.gov/nicpubweb/nicweb/HCSGreaterThan10B.aspx. Par ailleurs, la publication de l'OFR de 2015 sur les données de 2013, répertoriait déjà 33 institutions financières « sensibles ».

${ }^{21}$ Le groupe HSBC est considéré comme une Global Systemically Important Bank (G-SIB) sur le plan international et est domicilié au Royaume-Uni ; il n'est donc pas IFIS selon l'OFR car ce dernier utilise les données de HSBC North America Holdings Incorporation. Par ailleurs, la liste des IFIS au niveau global est divulguée tous les ans par le Financial Stability Board (voir Benoit et al. [2017b] pour une êtude sur la méthodologie développée par le comité de Bâle pour identifier les IFIS).
} 
Nous présentons dans la suite une application de notre méthodologie et une comparaison avec le classement réalisé par l'OFR en 2013, 2014 et 2015.

\section{Quelques résultats empiriques : Identifier et classer les institutions financières d'importance systémique}

Dans cette sous-section, nous appliquons notre méthodologie de classement des IFIS sur les différentes institutions américaines de notre base de données. Nous commençons tout d'abord par étudier les 8 institutions les plus « sensibles » qui sont mises en avant par le régulateur. Puis nous généralisons notre approche à l'ensemble des 60 potentielles IFIS américaines.

Dans le tableau 4 sont reportés les résultats des estimations du MEDAFS pour les 8 Global Systemically Important Bank (G-SIB) considérées. Le facteur de risque systémique ressort significatif pour l'ensemble des G-SIB et toutes voient leurs rentabilités impactées négativement par ce nouveau facteur. Le facteur de marché ressort également significatif tandis que les facteurs $S M B$ et $H M L$ ne le sont que pour la plupart des G-SIB (mais pas pour toutes).

Tableau 4 : Estimation du modèle à trois facteurs de Fama-French [1993] avec un facteur de risque systémique additionnel (ISRM) appliqué aux huit G-SIB américaines

Dans le tableau ci-dessous sont représentées les estimations du MEDAFS régit par l'équation :

$$
r_{i, t}=\alpha_{i}^{(I I)}+\beta_{i}^{(I I)} r_{M, t}+\theta_{i}^{(I I)} r_{S M B, t}+\gamma_{i}^{(I I)} r_{H M L, t}+\varphi_{i}^{(I I)} r_{I S R M, t}+\varepsilon_{i, t}^{(I I)}(I I) \text {. }
$$

\begin{tabular}{cllllll}
\hline \multicolumn{7}{c}{ MEDAFS (II) } \\
\hline \hline & $\alpha_{i}^{(I I)}$ & $\beta_{i}^{(I I)}$ & $\theta_{i}^{(I I)}$ & $\gamma_{i}^{(I I)}$ & $\varphi_{i}^{(I I)}$ & $\overline{R^{2}}$ \\
\hline JPM & 1,62 & $1,30^{* * *}$ & $-0,31^{* * *}$ & $-0,11^{* *}$ & $-2,76^{* * *}$ & $59,09 \%$ \\
BAC & $-2,16$ & $1,64^{* * *}$ & $-0,11^{* *}$ & $-0,05$ & $-3,17^{* * *}$ & $47,79 \%$ \\
MS & $-0,56$ & $1,88^{* * *}$ & 0,08 & $0,18^{* * *}$ & $-1,70^{* * *}$ & $52,54 \%$ \\
GS & 3,62 & $1,21^{* * *}$ & $-0,14^{* * *}$ & 0,03 & $-1,34 * * *$ & $54,87 \%$ \\
WFC & 1,34 & $1,28^{* * *}$ & $-0,25^{* * *}$ & $-0,34^{* * *}$ & $-2,98^{* * *}$ & $48,34 \%$ \\
BK & 0,64 & $1,35^{* * *}$ & $-0,05$ & $-0,35^{* * *}$ & $-1,69^{* * *}$ & $58,45 \%$ \\
STT & $-3,58$ & $1,47^{* * *}$ & $-0,09^{* *}$ & $-0,26^{* * *}$ & $-0,85^{* * *}$ & $47,74 \%$ \\
\hline
\end{tabular}

Source : Bloomberg, la base de données comprend un ensemble complet de prix des titres de notre base de données américaine comprenant 60 institutions financières en données quotidiennes du 03/09/2003 au 24/06/2014. Calculs des auteurs. Le tableau donne les valeurs des différents paramètres du MEDAF à trois facteurs de Fama et French [1993] avec prise en compte du risque systémique (modèle II) pour l'ensemble des 8 G-SIB listées par l'OFR. Les valeurs du paramètre $\alpha$ sont exprimées en $10^{-4}$. L'estimation des paramètres se fait par la méthode SURE de Zellner [1962] en système avec MCG. Les seuils de significativité sont fixés respectivement à $10 \%, 5 \%$ et $1 \%$ pour *, ** et ***. 
Le tableau 5 donne le nombre d'IFIS détectées pour un seuil de significativité donné selon deux méthodes d'estimation : les MCO et la méthode SURE de Zellner [1962]. Les différents seuils retenus sont : $0,1 \% ; 1 \% ; 5 \%$ et $10 \%$. Ces seuils, certes arbitraires, peuvent être adaptés aux conditions du marché et aux ressources des organismes de réglementation.

Dans cette proposition, un certain pourcentage des principales IFIS classées en fonction de l'importance de leurs sensibilités au risque systémique est fixé à $60 \%$ de manière à ne se focaliser que sur les plus systémiques. Plus la contrainte sur la significativité est lâche (passant donc de 0,1\% à 10\%), et, naturellement, plus le nombre d'IFIS détectées est important.

Par exemple, si le niveau de confiance est fixé à 0,1\% pour déterminer la significativité des sensibilités ${ }^{22}$ (seuil extrêmement sévère), et si le pourcentage des institutions à suivre est fixé à $60 \%$ des institutions significatives, alors 46 IFIS sont identifiées en utilisant les MCO et 32 pour la méthode SURE. Dans la dernière colonne du tableau 5, le niveau de confiance est fixé à 10\% pour déterminer la significativité des sensibilités (seuil peu sévère) : 54 (par les MCO) et 34 (en utilisant la méthode SURE) IFIS sont respectivement identifiées.

\section{Tableau 5 : Nombre d'IFIS détectées pour un seuil de significativité donné}

\begin{tabular}{|c|c|c|c|c|c|c|c|c|c|c|}
\hline & \multicolumn{2}{|c|}{$\begin{array}{c}\text { IFIS pour } \\
0,1 \% \text { de } \\
\text { significativité } \\
\end{array}$} & \multicolumn{2}{|c|}{$\begin{array}{c}\text { IFIS pour } \\
0,5 \% \text { de } \\
\text { significativité }\end{array}$} & \multicolumn{2}{|c|}{$\begin{array}{c}\text { IFIS pour } \\
1 \% \text { de } \\
\text { significativité }\end{array}$} & \multicolumn{2}{|c|}{$\begin{array}{c}\text { IFIS pour } \\
5 \% \text { de } \\
\text { significativité } \\
\end{array}$} & \multicolumn{2}{|c|}{$\begin{array}{c}\text { IFIS pour } \\
10 \% \text { de } \\
\text { significativité } \\
\end{array}$} \\
\hline Méthode d’Estimation & $\mathrm{MCO}$ & SURE & $\mathrm{MCO}$ & SURE & $\mathrm{MCO}$ & SURE & $\mathrm{MCO}$ & SURE & $\mathrm{MCO}$ & SURE \\
\hline $\begin{array}{c}\text { Nombre d'institutions } \\
\text { financières les plus } \\
\text { significatives } \\
\end{array}$ & 46 & 32 & 49 & 32 & 51 & 32 & 53 & 34 & 54 & 34 \\
\hline $\begin{array}{l}\text { Percentile des institutions } \\
\text { financières les plus } \\
\text { significatives (60\%) } \\
\end{array}$ & 27 & 19 & 29 & 19 & 30 & 19 & 31 & 20 & 32 & 20 \\
\hline $\begin{array}{l}\text { En pourcentage par } \\
\text { rapport à l'échantillon } \\
\text { global }\end{array}$ & $45 \%$ & $32 \%$ & $48 \%$ & $32 \%$ & $50 \%$ & $32 \%$ & $52 \%$ & $33 \%$ & $53 \%$ & $33 \%$ \\
\hline
\end{tabular}

Source : Bloomberg, la base de données comprend un ensemble complet de prix des titres de notre base de données composée de 60 institutions financières américaines en données quotidiennes du 03/09/2003 au 24/06/2014. Calculs des auteurs. Ce tableau donne le nombre d'IFIS détectées par notre méthodologie en fixant un percentile de $60 \%$ pour différents niveaux de significativité selon que l'estimation du modèle à trois facteurs de Fama-French [1993] étendu au risque systémique, se fait au moyen des MCO ou de la méthode SURE proposée par Zellner [1962]. Le tableau est constitué de 5 colonnes indiquant les seuils fixés à $0,1 \%, 0,5 \%, 1 \%$, 5\% et $10 \%$. La première ligne indique la méthode d'estimation, la deuxième indique le nombre d'institutions financières significatives, la troisième ligne indique le nombre d'institutions financières retenues à partir du percentile choisi (60\% ici) et la dernière ligne donne le pourcentage des institutions retenues sur l'ensemble des institutions.

\footnotetext{
${ }^{22}$ Il n’y a pas de différence entre les résultats obtenus avec des seuils de significativité fixés à $0,1 \%$ et à $1 \%$; une très faible et une faible pour les seuils à $5 \%$ et à $10 \%$ ( $C f$. tableau 5$)$.
} 
Concernant la comparaison de notre classement avec celui de l'OFR, l'objectif ici est de montrer que l'on peut aboutir à un classement des IFIS sans recourir à la méthodologie coûteuse et difficilement reproductible de l'OFR (du fait des données indisponibles au public). En outre, notre méthodologie n’implique pas le choix arbitraire relatif à la facette du risque systémique qui doit être considérée comme plus ou moins importante ; en effet, l'OFR fixe arbitrairement un poids équivalent au cinq caractéristiques du risque systémique qu'ils prennent en considération (la taille, l'interconnexion, la complexité, la substituabilité, l'activité juridictionnelle), alors que notre méthodologie s’appuie sur les données et peut-être, très facilement et à moindre coût, enrichie par l'ajout de nouvelles mesures, ou la prise en compte d'autres institutions, ou d'un secteur d'activité particulier comme le secteur de l'assurance.

La méthodologie de l'OFR a probablement comme avantage d'utiliser des données privées (qui élargissent le contenu informationnel de l'analyse, mais qui sont inaccessibles pour nous) au travers de 12 indicateurs regroupés en cinq catégories (avec des poids équipondérés), qui permettent d'obtenir un score systémique. Les désavantages se trouvent probablement dans la disponibilité des données et leur fréquence, et dans l'arbitrage établi au niveau de la contribution arbitraire au score final de chaque facette du risque systémique. En outre, un papier récent de Benoit et al. [2017] met en avant deux autres désavantages de la méthodologie utilisée par l'OFR, à savoir: le biais des classements au niveau de chaque catégorie et le problème du taux de change qui influence le classement des IFIS.

En résumé, notre méthodologie peut se comprendre comme plus flexible et plus transparente, et elle n'implique pas autant de contraintes. Enfin, nous ne fixons pas de poids arbitraires pour les facettes du risque systémique. Nous laissons tout simplement parler les données à notre disposition.

Dans le tableau 6, nous comparons les classements de l'OFR aux classements obtenus à partir du MEDAFS sur 3 années : 2013, 2014 et 2015. Le Panel A présente le classement de chaque $G$-SIB. Ce classement évolue au cours du temps, avec toutefois de fortes similarités entre les classements pour chaque institution financière selon les périodes. Plus précisément, le Panel B montre les corrélations de rang de Spearman $(\rho)$, Kendall $(\tau)$ et Pearson $(\gamma)$ sur ces 3 périodes pour les deux classements (OFR versus notre 
méthodologie). Ces différentes corrélations indiquent que, s'il existe une certaine proximité entre les deux classements, ils sont néanmoins différents.

Sur les trois périodes considérées (2013, 2014, 2015), les institutions JPM, CITIGROUP et BAC sont par exemple les plus systémiques pour l'OFR, tandis qu'elles occupent globalement les 2-ème, 3-ème et 4-ème places dans notre méthodologie. Nos classements sont donc assez proches des classements de l'OFR. Notre méthodologie capte par ailleurs la montée du risque systémique de WFC depuis 2013 qui, comme l’indique l'OFR sur son site ${ }^{23}$ et dans ses rapports ${ }^{24}$, accroît considérablement son risque. Les autres G-SIB sont plus ou moins classées dans le même ordre entre 2013 et 2015. Ainsi, les différences des classements et la comparaison avec ceux de l'OFR sont principalement dues aux facettes du risque systémique que chaque méthodologie met en avant.

\section{Tableau 6 : Comparaison des classements des 8 G-SIB américaines}

Panel A : Comparaison des classements sur trois périodes

\begin{tabular}{|c|c|c|c|c|c|c|}
\hline \multirow{2}{*}{$G-S I B$} & \multicolumn{3}{|c|}{ Classement effectué à partir de l’OFR } & \multicolumn{3}{|c|}{ Classement effectué à partir du MEDAFS } \\
\hline & 2013 & 2014 & 2015 & 2013 & 2014 & 2015 \\
\hline JPM & 1 & 1 & 1 & 3 & 3 & 4 \\
\hline CITIGROUP & 2 & 2 & 2 & 4 & 4 & 3 \\
\hline BAC & 3 & 3 & 3 & 2 & 2 & 2 \\
\hline MS & 4 & 5 & 6 & 8 & 6 & 7 \\
\hline GS & 5 & 4 & 4 & 6 & 1 & 6 \\
\hline WFC & 6 & 6 & 5 & 1 & 7 & 1 \\
\hline BK & 7 & 7 & 7 & 7 & 8 & 8 \\
\hline STT & 8 & 8 & 8 & 5 & 5 & 5 \\
\hline
\end{tabular}

Panel B : Corrélations avec le classement de l’OFR

\begin{tabular}{cccccccccc} 
& \multicolumn{3}{c}{2013} & \multicolumn{2}{c}{2014} & \multicolumn{2}{c}{2015} \\
\hline \hline Corrélations* & $\rho$ & $\tau$ & $\gamma$ & $\rho$ & $\tau$ & $\gamma$ & $\rho$ & $\tau$ & $\gamma$ \\
\hline Valeurs & 0,29 & 0,14 & 0,29 & 0,64 & 0,43 & 0,64 & 0,50 & 0,29 & 0,50
\end{tabular}

Source : Bloomberg, la base de données comprend un ensemble complet de prix des titres de notre base des 8 G-SIB en données quotidiennes du 03/09/2003 au 24/06/2014. Calculs des auteurs. Le Panel A donne les classements de l'OFR en 2013 en comparaison avec les classements obtenus par le modèle à trois facteurs de Fama-French [1993] étendu au risque systémique pour 3 périodes 2013, 2014 et 2015. Le Panel B renseigne sur les similarités des classements, au travers des corrélations entre ceux de l'OFR et ceux obtenus via le MEDAFS pour les 3 périodes $\left({ }^{*} \rho\right.$ : Spearman ; $\tau$ : Kendall ; $\gamma$ : Pearson).

\footnotetext{
${ }^{23}$ Cf. le graphique sur l'évolution des scores des 8 G-SIB américaines pour les années 2013, 2014 et 2015 est disponible sur: https://www.financialresearch.gov/gsib-scores-chart/

${ }^{24}$ Cf. l'OFR briefs d'avril 2016 : «...this brief shows that systemic importance scores rose significantly for three of the largest Chinese banks and Wells Fargo \& Co. In addition, U.S. banks continued to have the highest systemic importance scores. The scores show that many of the largest U.S. banks are highly interconnected and lack substitutes for the financial services they offer » (sic). Voir :

https://www.financialresearch.gov/briefs/files/OFRbr_2016-03_Systemic-Importance-Data-Shed-Lighton-Global-Banking-Risks.pdf
} 
Le graphique 4 représente l'évolution dynamique du classement de l’institution financière JPM (parmi les 8 autres G-SIB). On constate que ce classement évolue au cours du temps: JPM, par exemple, se situe entre la 2-ième et 8-ième place sur tout l'échantillon, avec un rang faible entre 2006 et 2007 (faible risque), qui se détériore rapidement durant la crise. Ceci illustre la nécessité de suivre dynamiquement ce classement à partir de notre méthodologie, car il est intuitif de penser que le classement des IFIS variera en fonction des conditions de marchés, du comportement individuel et collectif des institutions et de l'évolution du cadre de supervision réglementaire et du contrôle effectif des autorités.

\section{Graphique 4 : Evolution du classement de JPM}

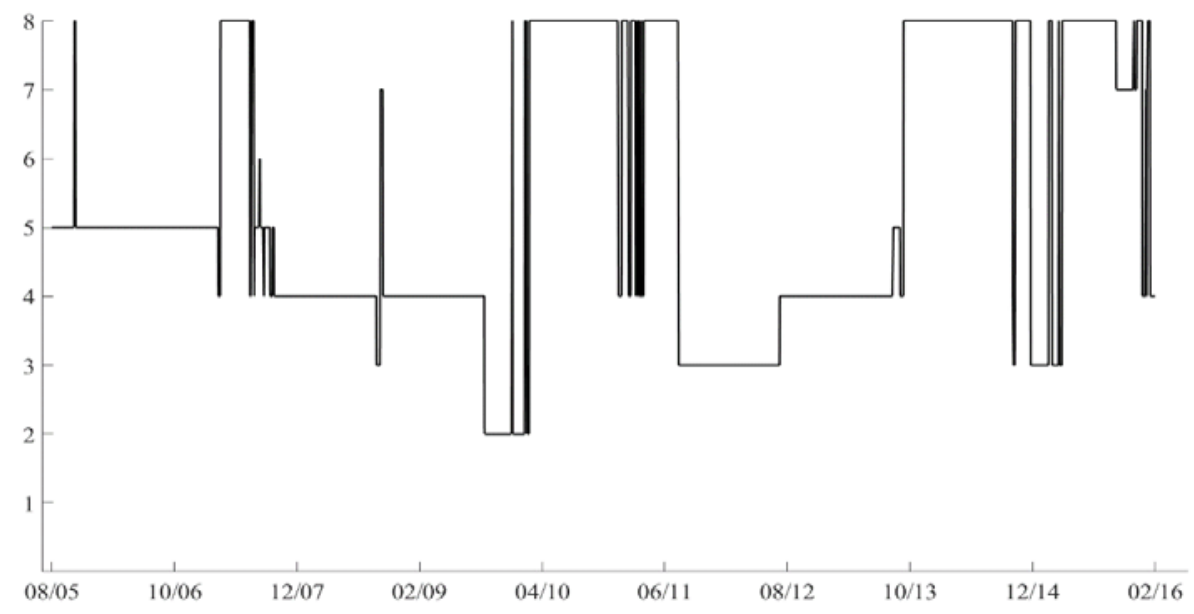

Source : Bloomberg, la base de données comprend un ensemble complet de prix des titres de notre base de données américaine comprenant 60 institutions financières en données quotidiennes du 2 septembre 2003 au 26 février 2016. Calculs des auteurs. Ce graphique représente l'évolution du classement de l'institution financière JPM sur l'ensemble de la période. En ordonnées sont représentées les positions qu'elle occupe dans le classement parmi les 8 G-SIB : du rang 1 (haut risque) au rang 8 (risque le plus faible des institutions les plus risquées).

Le graphique 5 donne la comparaison entre les IFIS désignées par l'OFR en 2013 et celles détectées en utilisant notre méthodologie pour un percentile fixé à $60 \%$ des IFIS classées par leurs sensibilités au risque systémique à un niveau de significativité fixé à 0,1\%. En gris foncé sont représentées le percentile des IFIS détectées par notre méthodologie (60\% ici, soit 32 institutions financières). En blanc sont représentées les institutions financières n’appartenant pas à ce percentile. Les barres en gris clair représentent les IFIS détectées seulement par l'OFR. Les huit G-SIB sont toutes détectées pour un percentile fixé à 60\%. Les bars en gris (mi- foncé, encadré de noir) représentent 
les IFIS détectées à la fois par l'OFR et par notre méthodologie sur la période du 03/09/2003 au 24/06/2014.

\section{Graphique 5 : Comparaison de la détection des IFIS}

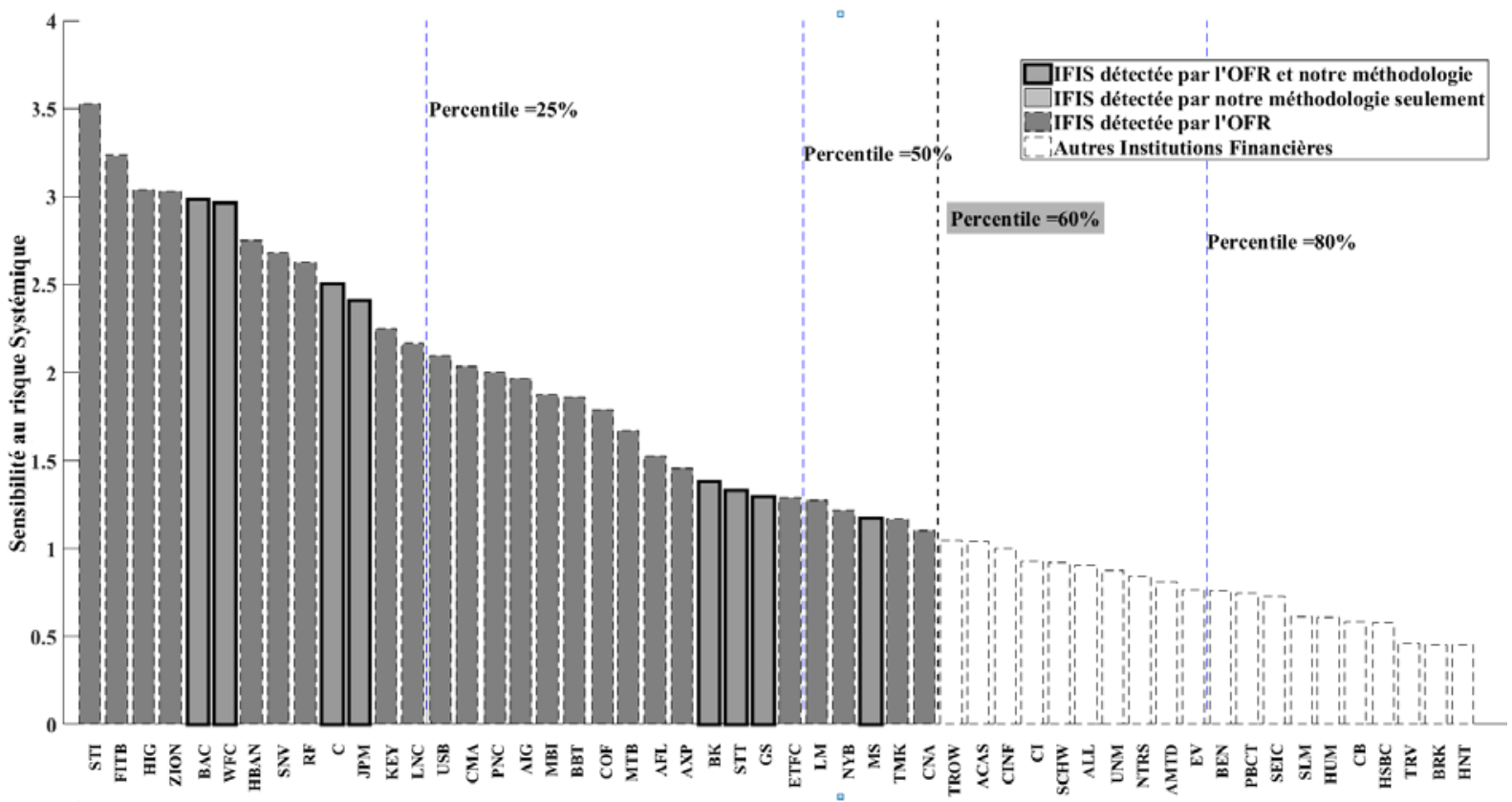

Source : Bloomberg, la base de données comprend un ensemble complet de prix des titres de notre base de données composée de 60 institutions financières américaines en données quotidiennes du 03/09/2003 au 24/06/2014. Calculs des auteurs. En abscisse sont reportés les Tickers des institutions financières et en ordonnées sont représentées les sensibilités (en valeur absolue) au risque systémique. On y retrouve également le classement obtenu par l'OFR en 2013 représenté par les bars de couleur gris clair, en gris foncé sont représentées les SIFI détectées par notre méthodologie (pour un percentile fixé à 60\% et un seuil de significativité à 0,1\%). En gris mi- foncé dont les bordures sont épaisses, sont représentées les SIFI détectées à la fois par notre méthodologie et par l'OFR. En blanc sont reportées les autres institutions financières. 


\section{Conclusion}

Nous avons ainsi dans cet article commencé par développer, à la suite de Kouontchou et al. [2017], une méthodologie de construction d'un indice de risque systémique, dénommé Index of Systemic Risk Measures (ISRM), calculé via une Analyse en Composantes Principales Parcimonieuses (ACPP) portant sur de nombreuses mesures de risque systémique (qui en capturent les différents aspects), afin de maximiser le lien potentiel entre la survenance d'un choc systémique et son impact sur la croissance future, de manière au final à ce que les actions entreprises pour réduire le risque systémique contribuent au mieux à limiter l'impact d'un tel choc sur l'économie réelle. La rationalité de l'exercice réside dans la multiplicité des métriques de risque systémique global introduites dans la littérature depuis la dernière crise financière globale, et des redondances potentielles entre celles-ci. Ces dernières émergent du fait que chaque métrique évalue une ou plusieurs facettes particulières du risque systémique, et qu'il nous faut prendre en considération le caractère multidimensionnel de ce risque global. Pour faire face à ce problème, une première approche consiste à organiser une comparaison relative des différentes approches et à déterminer, à l'aune de certains critères, la (les) meilleure(s) mesure(s) (e.g. Benoit et al. [2017a] et Poledna et al. [2017]). Une autre solution consiste à passer par une technique de réduction de l'information, qui se justifie aussi pleinement du fait de l'existence d'un important risque de modèle inhérent à leur estimation. A l'instar de Giglio et al. [2016] et de Kouontchou et al. [2017], c'est cette dernière voie que nous avons choisi de suivre et la méthodologie adoptée est basée sur une ACPP, qui permet de sélectionner un nombre plus ou moins réduit de mesures de risque systémique pour la construction de l'indice agrégé. En conséquence, l'indice ainsi obtenu est le plus parcimonieux possible et il a, par construction, une dynamique plus stable dans le temps. Sur l'ensemble de la période, les mesures qui contribuent le plus dans la construction de l'indice sont : le Spillover Index, le Herfindahl-Hirschman Index, la VaR, l'Absorption Ratio, la CoVaR, la MES, le Term Spread, le Default Yield Spread, la CES, la volatilité, le Dynamical Causality Index, la SRISK, le Turbulence Index, et la $\triangle C$ CoVaR. D’autres mesures viennent aussi s'ajouter dans sa composition, mais avec des poids relativement plus faibles. Les principales dimensions du risque systémique sont ainsi prises en compte dans notre indice final. 
Une fois cet indice construit, nous avons mis en évidence la relation entre le risque systémique et les rendements des actifs financiers à partir du Modèle d'Equilibre des Actifs Financiers (MEDAF), étendu à la Fama et French [1993], en ajoutant dans l'analyse le facteur de risque systémique précédemment construit. En incorporant ce nouveau facteur dans le MEDAF classique, nous montrons que le nouveau modèle d’équilibre, nommé Modèle d’Equilibre des Actifs Financiers avec risque Systémique (MEDAFS), permet de retrouver d'une part la relation classique entre les rendements de l'actif et le risque systématique (mesuré par le bêta), et, d'autre part, une relation entre les rendements de l'actif et le risque systémique. Nous montrons ainsi que le risque systémique influence significativement l'évaluation des actifs financiers.

Enfin, nous utilisons le MEDAFS pour identifier les Institutions Financières d'Importance Systémique (IFIS). Ainsi, un classement de ces institutions est proposé et effectué à partir des paramètres les plus statistiquement significatifs : l'importance des institutions financières serait ainsi déterminée par leur sensibilité (et leur significativité) au risque systémique global. Pour que le régulateur puisse concentrer ses forces, forcément limitées, sur les principales sources du risque systémique, nous proposons de fixer un percentile (60\% ici) des institutions financières ayant une sensibilité au risque systémique des plus grandes. Dans notre étude, nous identifions ainsi 32 IFIS parmi 54 institutions financières qui ont un risque systémique significatif. Par ailleurs, une comparaison avec le classement de l'OFR montre que les « Global Systemically Important Banks » américaines (US G-SIB) sont aussi identifiées au travers de notre proposition sur la période courant du 03/09/2003 au 24/06/2014.

Pour terminer, nous pouvons mentionner plusieurs pistes de recherche complémentaire qui s'ouvrent désormais.

En premier lieu, des recherches supplémentaires pourraient être menées concernant la construction de l'indice global de risque systémique. Il pourrait ainsi être intéressant d’essayer de compléter au mieux le panel de mesures de risque systémique utilisées dans la création de l'ISRM, de manière à ne sous-estimer aucun aspect du risque systémique. Par exemple, d'autres mesures utilisant des données de marchés pourraient permettre d'améliorer l'approche proposée, en considérant par exemple la Systemic Expected Shortfall (SES) d'Acharya et al. [2017] dont les auteurs en ont montré le pouvoir prédictif sur l'émergence du risque systémique durant la crise de 2007-2009, ou encore le Realized 
Systemic Risk Beta (RSRB) de Hautsch et al. [2015] qui permettrait de mieux mesurer la contribution des IFIS à un événement systémique au travers de leurs expositions et de leurs interconnexions. Le fait que nous utilisions une ACPP et un critère de pertinence relatif au lien entre risque systémique et économie réelle, nous permet de gérer les potentielles redondances de chacune des nouvelles mesures prises en considération, et, au final, de juger l'apport de telle ou telle mesure supplémentaire dans l'analyse globale.

En second lieu, plusieurs prolongements concernant le MEDAFS pourraient être envisagés. Afin de vérifier plus avant la robustesse du MEDAFS, il serait pertinent d'étendre cette étude sur d'autres marchés, plus larges (comme le DJI500 aux USA et le SBF120 en France). D’autres extensions du MEDAF original sont également envisageables, avec l'ajout de facteurs supplémentaires (Brown [1989] ; Jegadeesh et Titman [1993] ; Carhart [1997] ; Pástor et Stambaugh [2003] ; Fama et French, [2012] et [2015] ; Holderness et Pontiff [2016] ; Feng et al. [2017]), au risque peut-être de considérer des variables très corrélées entre elles. ${ }^{25}$ Concernant la méthode d’estimation, des améliorations sur la méthode SURE de Zellner [1962] sont à l'étude, au travers par exemple de la prise en compte de l'incertitude sur la matrice de variance-covariance des Moindres Carrés Généralisés (MCG), et le remplacement du calcul matriciel dans la méthode SURE utilisant des produits de Kronecker, par une réécriture des programmes de minimisation en utilisant des tenseurs et des produits d'Hadamard. Il s'agirait là de décomposer chaque résultante des différents produits impliqués dans le calcul, afin de le rendre plus efficace.

En troisième lieu enfin, notre proposition de méthodologie pour l'identification des IFIS pourrait certainement être améliorée encore grâce à l'utilisation de modèles de prévision des différentes variables sous-jacentes entrant dans la construction d'un système d'alerte (Early Warning System ; e.g. Candelon et al. [2012] ; [2014]) de manière à anticiper au mieux les évolutions du système global et le comportement des principaux acteurs de marché.

\footnotetext{
${ }^{25}$ Carhart [1997] propose par exemple de rajouter l'effet momentum représentant la différence entre les rendements des titres performants (Winners) et ceux des titres les moins performants (Losers) sur le marché comme facteur supplémentaire (noté $W M L$ ). Toutefois, le facteur momentum et notre facteur de risque systémique, bien que construits différemment, montrent un coefficient de corrélation de 0,70 sur toute la période étudiée (et de 0,90 sur la période de crise 2008-2009). L'intuition étant que les turbulences de marché s’accompagnent en général de primes de risques négatives sur les marchés actions.
} 


\section{Références}

Abergel F., Chakrabarti B., Chakraborti A. et GHOSh A. [2013], Econophysics of Systemic Risk and Network Dynamics, Springer, 298 pages.

Acharya V., Pedersen L., Philippon T. et Richardson M. [2017], « Measuring Systemic Risk », Review of Financial Studies, 30 (1), p. 2-47.

Acharya V., Brownless C., Engle R., Farazmand F. et Richardson M. [2013], " Measuring Systemic Risk », dans Roggi-Altman (dir.), Managing and Measuring Risk: Emerging Global Standards and Regulation after the Financial Crisis, World Scientific Series in Finance, p. 65-98.

Acharya V., Engle, R. ET Richardson M. [2012], « Capital Shortfall: A New Approach to Ranking and Regulating Systemic Risks ", American Economic Review, 102, p. 59-64.

Adrian T. et Brunnermeier M. [2016], « CoVaR », American Economic Review, 106 (7), p. 1705-1741.

Allen L., Bali T. et Tang Y. [2012], « Does Systemic Risk in the Financial Sector Predict Future Economic Downturns? », Review of Financial Studies, 25 (10), p. 3000-3036.

Aminud Y. [2002], " Illiquidity and Stock Returns: Cross-section and Time-series Effects », Journal of Financial Markets, 5, p. 31-56.

Banulescu G. eT Dumitrescu E. [2015], «Which are the SIFIs? A Component Expected Shortfall Approach to Systemic Risk », Journal of Banking and Finance, 55, p. 575-588.

Barone-Adesi G., Gagliardini P. et Urga G., [2003], « Testing Asset Pricing Models with Coskewness », Journal of Business and Economic Statistics, 22 (4), p. 474485.

Benoit S., Colliard J.-E., Hurlin C. et Perignon C. [2017a], « Where the Risks Lie: A Survey on Systemic Risk », Review of Finance, 21 (1), p. 109-152.

Benoit S., Hurlin C. et Perignon C. [2017b], " Pitfalls in Systemic-risk Scoring », HEC Paris Research Paper, FIN-2013-1005, 73 pages.

Billio M., Getmansky M., Lo A. Et Pelizzon L. [2012], « Econometric Measures of Connectedness and Systemic Risk in the Finance and Insurance Sectors », Journal of Financial Economics, 104 (3), p. 535-559.

Billio M., Caporin M., Panzica R. ET Pelizzon L. [2017], « The Impact of Network Connectivity on Factor Exposures, Asset Pricing and Portfolio Diversification », SAFE Working Paper 166, 63 pages.

Bisias D., Flood M., Lo A. et Valavanis S. [2012], "A Survey of Systemic Risk Analytics », Annual Review of Financial Economics, 4, p. 255-296.

Black F., Jensen M. et Scholes M. [1972], « The Capital Asset Pricing Model: Some Empirical Tests », dans Studies in the Theory of Capital Markets, Praeger Publishers Inc., 54 pages.

Bongini P., Nieri L. ET Pelagatti M. [2015], « The Importance of being Systemically Important Financial Institutions », Journal of Banking and Finance, 50, p. 562-574. 
Boucher C., Daníelsson J., Kouontchou P. et Maillet B. [2014], « Risk Models-atRisk », Journal of Banking and Finance, 44, p. 72-92.

Boucher C., Kouontchou P. et Maillet B. [2016], «Du Risque des Mesures de Risque Systémique », Revue Economique, 2 (67), p. 263-278.

BRown S. [1989], « The Number of Factors in Security Returns », Journal of Finance, 44, p. 1247-1262.

BRownlees C. eT ENGLE R. [2017], « SRISK: A Conditional Capital Shortfall Index for Systemic Risk Measurement », Review of Financial Studies, 30 (1), p. 48-79.

Candelon B., Dumitrescu E. ET Hurlin C. [2014], « Currency Crises Early Warning Systems: Why they should be Dynamic », International Journal of Forecasting, 30 (4), p. 1016-1029.

CAndelon B., Dumitrescu E. et Hurlin C. [2012], « How to evaluate an Early Warning System? ToArds a United Statistical Framework for Assessing Financial Crises Forecasting Methods », IMF Economic Review, 60 (1), p. 75-113.

CARHART M. [1997], « On Persistence in Mutual Fund Performance », Journal of Finance 52, p. 57-82.

Chen L., Collin-Dufresne P. et Goldstein R. [2009], « On the Relation between Credit Spread Puzzles and the Equity Premium Puzzle ", Review of Financial Studies, 22 (9), p. 3367-3409.

Chen N.-F., Roll R. et Ross S. [1986], " Economic Forces and the Stock Market », Journal of Business, 59 (3), p. 383-403.

Cochrane J. [2011], « Presidential Address: Discount Rates », Journal of Finance, 66, p. 1047-1108.

Cont R. et Wagalath L. [2013], " Running for the Exit: Distressed Selling and Endogenous Correlation in Financial Markets ", Mathematical Finance, 4 (23), p. 718-741.

DANíELSSON J. [2002], « The Emperor has no Clothes: Limits to Risk Modelling », Journal of Banking and Finance, 26, p. 1273-1296.

Danielsson J., James K., VAlenZuela M. Et Zer I. [2015], « Model Risk of Systemic Risk Models », Journal of Financial Stability, 23, p. 79-91.

DAS S. [2016], « Matrix Metrics: Network-Based Systemic Risk Scoring », Journal of Alternative Investments, 18 (4), p. 33-51.

De Bandt O., Heam J.-C., Labonne C. et Tavolaro S. [2015], « La Mesure du Risque Systémique après la Crise Financière », Revue Economique, 66 (3), p. 481-500.

Diebold F. ET Yilmaz K. [2014], « On the Network Topology of Variance Decompositions: Measuring the Connectedness of Financial Firms », Journal of Econometrics, 182 (1), p. 119-134.

Diebold F. et Yilmaz K. [2009], « Measuring Financial Asset Return and Volatility Spillovers, with Application to Global Equity Markets », Economic Journal, 119, p. 158-171.

Estrella A. et Trubin M. [2006], « The Yield Curve as a Leading Indicator: Some Practical Issues », Current Issues in Economics and Finance, 12 (5), p. 1-7.

FAma E. ET French K. [2015], « A Five-Factor Asset Pricing Model », Journal of Financial Economics, 116, p. 1-22. 
FAma E. ET FREnch K. [2012], « Size, Value, and Momentum in International Stock Returns », Journal of Financial Economics, 105, p. 457-472.

FAMA E. ET FRENCH K. [1996], « Multifactor Explanations of Asset Pricing Anomalies », Journal of Finance, 51, p. 55-84.

FAMA E. ET FrenCH K. [1993], "Common Risk Factors in the Returns on Stocks and Bonds ", Journal of Financial Economics, 33, p. 3-56.

FAmA E. ET FRENCH K. [1992], « The Cross-section of Expected Stock Returns », Journal of Finance, 47, p. 427-465.

Feng G., Giglio S. ET XiU D. [2017], «Taming the Factor Zoo », Chicago Booth Research Paper, $N^{\circ} 17-4,69$ pages.

Galagedera D. ET MAHARAJ E. [2008], «Avelet Time-scales and Conditional Relationship between Higher-order Systematic Co-moments and Portfolio Returns », Quantitative Finance, 8 (2), p. 201-215.

Giglio S., Kelly B. et PruitT S. [2016], « Systemic Risk and the Macroeconomy: An Empirical Evaluation », Journal of Financial Economics, 119 (3), p. 457-471.

GilChrist S. ET ZAKRAJSEK E. [2012], «Credit Spreads and Business Cycle Fluctuations », American Economic Review, 102 (4), p. 1692-1720.

Green W. [2012], « Econometric Analysis », Pearson, 7 $7^{\text {th }}$ Edition, 1241 pages.

HANSON S., KASHYAP A. ET STEIN J. [2011], « A Macroprudential Approach to Financial Regulation », Journal of Economic Perspectives, 25 (1), p. 3-28.

Harvey C., LiU Y. ET Zhu H. [2016], «... and the Cross Section of Expected Returns », Review of Financial Studies, 29 (1), p. 5-68.

Hastie T., TibshiRAni R. ET Ainwright M. [2015], Statistical Learning with Sparsity: The Lasso and Generalizations, dans Chapman \& Hall/CRC Monographs on Statistics \& Applied Probability 143, 367 pages.

Hautsch N., Schaumburg J. Et Schienle M. [2015], « Financial Network Systemic Risk Contributions », Review of Finance, 19 (2), p. 685-738.

Holderness C. ET PonTIFF J. [2016], « Does Academic Research destroy Stock Return Predictability? », Journal of Finance, 71 (1), p. 5-32.

HoNG Y., LIU Y. ET ANG S. [2009], « Granger Causality in Risk and Detection of Extreme Risk Spill-over between Financial Markets », Journal of Econometrics, 150 (2), p. 271-287.

JegadeEsh N. ET TitMAn S. [1993], « Returns to Buying Winners and Selling Losers: Implications for Stock Market Efficiency », Journal of Finance, 48 (1), p. 65-91.

Kouontchou P., Maillet B., Modesto A. Et TokPavi S. [2017], «Quand l’Union fait la Force : un Indice de Risque Systémique », à paraître dans Revue Economique, 24 pages.

Kraus A. et LitZenberger R. [1976], « Skewness Preferences and the Valuation of Risk Assets », Journal of Finance, 31 (4), p. 1085-1100.

KRITZMAn M. ET Li Y. [2010], « Skulls, Financial Turbulence, and Risk Management », Financial Analysts Journal, 66 (5), p. 30-41.

Kritzman M., Li Y., Page S. et Rigobon R. [2011], " Principal Components as a Measure of Systemic Risk », Journal of Portfolio Management, 37 (4), p. 112-126. 
KuPIEC P. et GunTay L. [2015], « Testing for Systemic Risk using Stock Returns », AEI Economic Working Paper 2015-02, 52 pages.

LE PAN N. [2016], « Fault Lines: Earthquakes, Insurance, and Systemic Financial Risk », C.D. HOWE Institute, Commentary 454, 24 pages.

LINTNER J. [1965], « The Valuation of Risk Assets and the Selection of Risky Investments in Stock Portfolios and Capital Budgets », Review of Economics and Statistics, 47, p. 13-37.

Moenninghoff S., Ongena S. Et Wieandt A. [2015], « The Perennial Challenge to Abolish Too-Big-To-Fail in Banking: Empirical Evidence from the New International Regulation dealing with Global Systemically Important Banks ", Journal of Banking and Finance, 61, p. 221-236.

Moreno M. et PeÑA J. [2013], « Systemic Risk Measures: The Simpler the Better? », Journal of Banking and Finance, 37, p. 1817-1831.

Mossin J. [1966], « Equilibrium in a Capital Asset Market », Econometrica, 34 (4), p. 768-783.

Newey W. et West K. [1987], « A Simple, Positive Semi-definite, Heteroskedasticity and Autocorrelation Consistent Covariance Matrix », Econometrica, 55 (3), p. 70308 .

Nucera F., SchAab B., Koopman S.J. et Lucas A. [2016], "The Information in Systemic Risk Rankings », Journal of Empirical Finance, 38, p. 461-475.

PÁstor L. ET Stambaugh R. [2003], « Liquidity Risk and Expected Stock Returns », Journal of Political Economy, 111 (3), p. 642-685.

Poledna S., Bochmann O. eT Thurner S. [2017], « Basel III Capital Surcharges for GSIBs are far less Effective in Managing Systemic Risk in comparison to NetworkBased, Systemic Risk-dependent Financial Transaction Taxes », Journal of Economic Dynamics and Control, 77, p. 230-246.

Ross S. [1976], « The Arbitrage Theory of Capital Asset Pricing », Journal of Economic Theory, 13, 341-360.

Sharpe W. [1964], « Capital Asset Prices: A Theory of Market Equilibrium under Conditions of Risk », Journal of Finance, 19 (3), p. 425-442.

STEWART G. [1987], "Collinearity and Least Squares Regression », Statistical Science, 2, p. 68-100.

TIBSHIRANi R. [1996], " Regression Shrinkage and Selection via the LASSO », Journal of the Royal Statistical Society, Series B (Methodological), 58 (1), p. 267-288.

Ueda K. ET Weder di Mauro B. [2013], « Quantifying Structural Subsidy Values for Systemically Important Financial Institutions », Journal of Banking and Finance, 37 (10), p. 3830-3842.

White H. [1980], « A Heteroskedasticity-Consistent Covariance Matrix Estimator and a Direct Test for Heteroskedasticity », Econometrica, 48 (4), p. 817-838.

ZELLNER A. [1962], "An Efficient Method for estimating Seemingly Unrelated Regressions and Tests for Aggregation Bias », Journal of the American Statistical Association, 57 (298), p. 348-368.

Zou H., Hastie T. et Tibshirani R. [2006], « Sparse Principal Component Analysis », Journal of Computational and Graphical Statistics, 15 (2), p. 265-286. 


\section{Annexes.}

\section{Variables, base de données, méthodes, modèles et relation risque systématique- systémique}

Dans cette annexe, nous fournissons les mesures de risque systémiques utilisées dans la construction de l'indice de risque systémique (Section A.1), sur un ensemble d'institutions financières américaines (Section A.2). Nous renvoyons à l'article de Kouontchou et al. [2017] pour la définition des mesures, ainsi qu'aux auteurs des mesures, et nous ajoutons la définition de la Value-at-Risk, de la CES de Banulescu et Dumitrescu [2015], de la volatilité agrégée et de la SRISK de Acharya et al. [2012] et Brownlees et Engle [2017]. La section suivante présente la matrice des corrélations (inconditionnelles et conditionnelles) entre ces différentes mesures de risque systémique (Section A.3). Puis, dans la suite, nous détaillons les méthodologies utilisées dans cet article : nous détaillons la construction de l'indice de risque systémique à partir de la méthode ACPP (Section A.4). Indice qui est utilisé comme facteur supplémentaire dans le MEDAF traditionnel étendu au modèle à trois facteurs de Fama et French [1993] conduisant au MEDAF étendu au risque systémique (Section A.5) ; les estimations du nouveau modèle sont faites à partir de la méthode SURE (pour Seemingly Unrelated Regressions Estimation) proposée par Zellner [1962]. Nous présentons (Section A.6) brièvement un résumé du modèle de représentation des liens entre risque systémique et risque systématique tel qu’illustré dans Billio et al. [2017]. Enfin, la dernière section (Section A.7) est dédiée à l'évolution des coefficients de sensibilité au risque systématique et au risque systémique pour l’institution financière JPM.

\section{Annexe A.1. Mesures de risque systémique}

Dans la construction de l'Index of Systemic Risk Measures (ISRM), nous utilisons les mesures de risque systémiques suivantes afin de capturer l'instabilité, l'activité économique, le degré d'interconnexion et de concentration et les conditions de liquidité 
dans le système (Cf. Giglio et al. [2016] et Kouontchou et al. [2017]). Ces mesures peuvent être classées en quatre catégories :

- Les variables macro financières utilisées comme indicateurs avancés de l'activité économique : Credit Default Yield Spread, TED Spread and Term Spread.

- Agrégation des mesures de risque systémique entre les institutions financières : la Valueat-Risk conditionnelle (CoVaR) et la Delta Conditional Value-at-Risk( $\triangle \mathrm{CoVaR})$ d'Adrian et Brunnermeier [2016] et la Marginal Expected Shortfall (MES) de Acharya et al. [2013] et Brownlees et Engle [2017].

- Les mesures de risque systémique utilisées pour prendre en compte le degré d'interconnexion et la concentration dans le système : l'indice Spillover de Diebold et Yilmaz [2009], l'indice de causalité dynamique (DCI) de Billio et al. [2012], la mesure Turbulence de Kritzman et Li [2010], l’Absorption Ratio (AR) de Kritzman et al. [2011] et l'indice de concentration de Herfindahl-Hirschman.

- Nous utilisons aussi la mesure Amihud Illiquidity Measure (AIM) d'Amihud [2002] pour prendre en compte les conditions de liquidité dans le système.

Ces 16 mesures correspondent dans l'ordre à : $M_{1}$ : Spillover Index, $M_{2}:$ HerfindahlHirschman Index, $M_{3}:$ VaR, $M_{4}:$ Absorption Ratio, $M_{5}:$ CoVaR, $M_{6}:$ MES, $M_{7}:$ Term Spread, $M_{8}$ : Default Yield Spread, $M_{9}$ : CES, $M_{10}$ : Volatility, $M_{11}$ : Dynamical Causality Index, $M_{12}$ : SRISK, $M_{13}$ : Turbulence Index, $M_{14}: \triangle C o V a R, M_{15}:$ TED Spread et $M_{16}$ : Amihud Illiquidity Measure. Nous ne présentons ici que les quatre mesures additionnelles à l'ensemble des douze mesures utilisées par Kouontchou et al. [2017].

- $\quad$ Mesure $3\left(M_{3}\right)$, Value-at-Risk (VaR) : il s’agit ici de la valeur en risque du système financier (ou de manière pratique d'un indice de marché), correspondant à une perte maximale potentielle qui ne devrait être atteinte qu'avec une probabilité donnée sur un horizon temporel donné :

$$
\operatorname{Pr}\left(r_{i, t} \leq \operatorname{VaR}_{i, t}(\alpha)\right)=\alpha,
$$

avec $r_{i, t}$ les rendements de l'institution $i$ à la date $t$ pour un seuil de risque $\alpha$. 
- Mesure 9 (M9), Component Expected Shortfall (CES) : est une mesure proposée par Banulescu et Dumitrescu [2015] ; elle quantifie la contribution d'une institution financière au risque du système en multipliant la $M E S_{i, t}$ de l'institution $i$ à la date $t$ par le poids de cette même institution au sein du système :

$$
C E S_{i, t}(\alpha)=-w_{i, t} M E S_{i, t}\left(\alpha=E\left(r_{i, t} \mid r_{m, t} \leq \operatorname{VaR}_{m, t}(\alpha)\right)\right)
$$

avec $r_{i, t}, r_{m, t}$ les rendements de l'institution $\boldsymbol{i}$ et du marché et $\operatorname{VaR}_{m, t}(\alpha)$ la $\operatorname{VaR}$ du portefeuille de marché pour un seuil de risque $\alpha$. Le poids $w_{i, t}$ de l'institution financière $i$ correspond au rapport de sa capitalisation boursière sur la somme totale des capitalisations boursières des institutions au sein du système à la date $t$.

- Mesure 10 (M10), Volatility (Vol) qui est l'agrégation des volatilités des institutions financières au sein du marché. Elle est définie comme étant l'écart type des rentabilités journalières sur une période d’une année en jours ouvrés.

- Mesure 12 (M12), Systemic RISK (SRISK) : construite par Acharya et al. [2012] et Brownlees et Engle [2017], elle correspond au montant de capital requis par une institution financière en situation de stress quand le marché est en même temps en situation de troubles :

$$
\operatorname{SRISK}_{i, t}(1-\alpha)=\max \left\{0, \gamma D_{i, t}-(1-\gamma) W_{i, t}\left[1-\operatorname{LRMES}_{i, t}(1-\alpha)\right]\right\},
$$

avec $\gamma$ le ratio de prudential capital requirement exigé par le régulateur, $D_{i, t}$ le montant de dette de l'institution $i$ à la date $t$ et $W_{i, t}$ le montant des capitaux propres de l'institution $i$ à la date $t$. La $\operatorname{LRMES}_{i, t}(1-\alpha)$ correspond à l'approximation de long terme de la $M E S_{i, t}(1-\alpha)$ de l'institution $i$ à la date $t$ et est définie comme suit :

$$
\operatorname{LRMES}_{i, t}(1-\alpha) \approx 1-\exp \left[18 \times M E S_{i, t}(1-\alpha)\right] .
$$




\section{Annexe A.2. La base de données des institutions financières}

A partir d'une base de données qui contient les valeurs nettes des actifs et les caractéristiques des 95 (grandes) institutions financières (Cf. Brownlees et Engle, [2017]), nous construisons une base de données cylindrique des valeurs nettes en USD pour 60 institutions financières du 03/09/2003 au 26/02/2016, dont la liste nominative est présentée dans le tableau suivant.

Tableau A.2.1 : Noms et abréviations des Institutions Financières considérées

\begin{tabular}{|c|c|c|c|c|c|c|c|}
\hline \multicolumn{2}{|r|}{ Banques (21) } & \multicolumn{2}{|r|}{ Assurance (20) } & \multicolumn{2}{|c|}{ Courtiers (5) } & \multicolumn{2}{|r|}{ Autres (14) } \\
\hline BAC & Bank of America & $\begin{array}{l}\text { AFL } \\
\text { AIG }\end{array}$ & $\begin{array}{l}\text { Aflac } \\
\text { American International }\end{array}$ & ETFC & E-Trade Financial & ACAS & American Capital \\
\hline BBT & $\mathrm{BB} \& \mathrm{~T}$ & & Group & GS & Goldman Sachs & AMTD & TD Ameritrade \\
\hline BK & Bank of New York Mellon & ALL & Allstate Corp & MS & Morgan Stanley & AXP & American Express \\
\hline $\mathrm{C}$ & Citigroup & AOC & Aon Corp & SCHW & SchAb Charles & BEN & Franklin Resources \\
\hline CMA & Comerica Inc. & BRK & Berkshire HathaAy & TROW & T. Rowe Price & BLK & Blackrock \\
\hline HBAN & $\begin{array}{l}\text { Huntington Bancshares } \\
\text { Hong Kong \& Shanghai }\end{array}$ & CB & Chubb Corp & & & $\mathrm{COF}$ & Capital One Financial \\
\hline HSBC & Banking Corporation & CI & CIGNA Corp & & & EV & Eaton Vance \\
\hline JPM & JP Morgan Chase & CINF & Cincinnati Financial Corp & & & FITB & Fifth Third Bancorp \\
\hline KEY & Keycorp & CNA & CNA Financial corp & & & FNM & Fannie Mae \\
\hline МТВ & M \& T Bank Corp & HIG & Hartford Financial Group & & & FRE & Freddie Mac \\
\hline NTRS & $\begin{array}{l}\text { Northern Trust } \\
\text { New York Community }\end{array}$ & HNT & Health Net & & & HRB & H\&R Block \\
\hline NYB & Bancorp & HUM & Humana & & & $\mathrm{LM}$ & Legg Mason \\
\hline PBCT & Peoples United Financial & LNC & Lincoln National & & & SEIC & SEI Investments Company \\
\hline PNC & PNC Financial Services & MBI & MBIA & & & SLM & SLM Corp \\
\hline RF & Regions Financial & MMC & Marsh \& McLennan & & & & \\
\hline SNV & Synovus Financial & PGR & Progressive & & & & \\
\hline STI & Suntrust Banks & TMK & Torchmark & & & & \\
\hline STT & State Street & TRV & Travelers & & & & \\
\hline USB & US Bancorp & $\mathrm{UNH}$ & United health Group & & & & \\
\hline WFC & Wells Fargo \& Co & UNM & Unum Group & & & & \\
\hline ZION & Zion & & & & & & \\
\hline
\end{tabular}

Source : Bloomberg. Dans ce tableau sont reportés les Tickers des institutions. 


\section{Annexe A.3. Table des corrélations entre les mesures de risque systémique}

Le tableau suivant donne les corrélations de Pearson et de Spearman entre les mesures de risque systémique représentées dans le graphique 1 ( $C f$. article). Les corrélations supérieures à 0.80 sont en gras.

Tableau A.3.1 : Corrélation de Pearson et de Spearman entre les mesures de risque systémique

\begin{tabular}{|c|c|c|c|c|c|c|c|c|c|c|c|c|c|c|c|c|}
\hline & $M_{1}$ & $M_{2}$ & $M_{3}$ & $M_{4}$ & $M_{5}$ & $\boldsymbol{M}_{6}$ & $M_{7}$ & $M_{8}$ & $M_{9}$ & $M_{10}$ & $M_{11}$ & $M_{12}$ & $M_{13}$ & $M_{14}$ & $M_{15}$ & $M_{16}$ \\
\hline$M_{1}$ & 1,00 & 0,43 & 0,59 & & 0,60 & & 0,45 & & & 0,61 & $-0,23$ & 0,84 & 0,23 & 0,66 & 0,26 & \\
\hline$M_{2}$ & 0,40 & 1,00 & 0,38 & 41 & 0,43 & 035 & 068 & 0,39 & & 0,51 & & 0,47 & $-0,11$ &, 35 & $-0,19$ & 0,60 \\
\hline$M_{3}$ & 0,79 & 0,36 & 1,00 & 37 & 0,94 & 0.97 & 0,31 & 0,87 & 7 & 0,79 & 0,11 & 0,59 & 0,52 & 0,93 & 0,67 & 0,57 \\
\hline$M_{4}$ & 0,72 & 0,30 & 0,48 & 1,00 & 0,31 & 0,36 & 0,54 & 0,34 & 0,37 & 0,37 & 24 & 0,83 & 09 & 0,42 & 0,06 & 0,45 \\
\hline$M_{5}$ & 0,67 & 0,35 & 0,77 & 0,23 & 1,00 & 0,92 & 0,33 & 0,89 & & 82 & & 0,50 & 45 & & 0,57 & 0,62 \\
\hline$M_{6}$ & 0,72 & 0,26 & 0,91 & 0,4 & 0,71 & 1,00 & 0,24 & 0,88 & 0 & 0,72 & 0,13 & 0,60 & 0,52 & 0,94 & 0,69 & 0,49 \\
\hline$M_{7}$ & 0,32 & 0,63 & 0,44 & 0,33 & 0,38 & 0,26 & 1,00 & 0,1 & 4 & 0,47 & $-0,01$ & 0,44 & $-0,09$ & 0,25 & $-0,11$ & 0,54 \\
\hline$M_{8}$ & 0,71 & 0,29 & 0,70 & 0,32 & 0,68 & 0,66 & 0,08 & 1,00 & $0,8 /$ & 0,74 & 0,09 & 0,56 & 0,40 & $0,0 J$ & 0,55 & 0,52 \\
\hline$M_{9}$ & 0,73 & 0,25 & 0,89 & 0,45 & 0,71 & 0,99 & 0,2 & 0,6 & 1,0 & 0,7 & & & 0,53 & & 0,69 & 0,48 \\
\hline$M_{10}$ & & 0,47 & & 0,4 & 0,67 & 0,74 & 0,61 & & 0,72 & 1,00 & & & 0,16 & & 0,28 & 0,93 \\
\hline$M_{11}$ &, 21 & & $-0,23$ & & 0,01 & $-0,13$ & & $-0,21$ & & $-0,32$ & & $-0,31$ & ,20 & & 0,21 & $-0,22$ \\
\hline$M_{12}$ & & 0,38 & & & 0,40 & & & & & 0,74 & & & & &, 16 & 0,56 \\
\hline$M_{13}$ & 0,14 & $-0,24$ & & & 0,22 & 0,23 & $-0,28$ & & 0,22 & $-0,05$ & & 0,02 & 1,00 & & 0,65 & $-0,05$ \\
\hline$M_{14}$ & & & & & 0,85 & & U & & & 0,67 & $-0,02$ & &, 27 & & 0,68 & 0,43 \\
\hline$M_{15}$ & 0,08 & $-0,39$ & 0,15 & $-0,19$ & 0,34 & 0,26 & $-0,49$ & 0,4 & 0,26 & $-0,03$ & 0,17 & $-0,01$ & 0,48 & & 1,00 & 0,00 \\
\hline$M_{16}$ & 0,76 & 0,64 & 0,75 & 0,64 & 0,46 & 0,61 & 0,63 & 0,54 & 0,60 & 0,88 & $-0,39$ & 0,84 & $-0,20$ & 0,54 & $-0,29$ & 1,00 \\
\hline \multicolumn{17}{|c|}{ 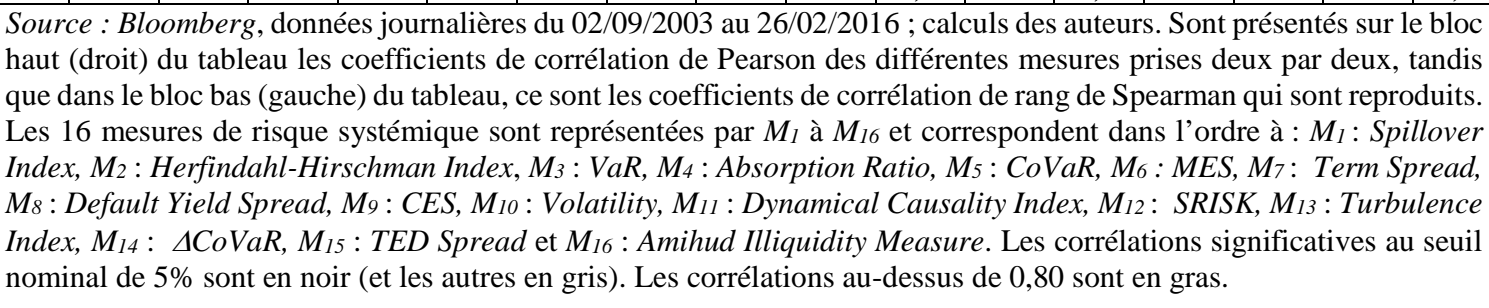 } \\
\hline
\end{tabular}

L’analyse des corrélations conditionnelles entre les différentes mesures de risque, i.e. les corrélations au-dessus du quantile à $\alpha=80,00 \%$ (pics simultanés deux à deux des mesures de risque systémique) sont toutes égales à 1,00 et significatives au seuil de $1,00 \%$. 


\section{Annexe A.4. La méthode ACPP}

Du point de vue statistique, si nous considérons une matrice $M$ de dimension $(T, p)$ des z-scores des données initiales - ici nos mesures de risque systémique (Cf. Benoit et al. [2017b]), la première composante principale (premier axe), notée $x$, est un vecteur de dimension $p$ solution du programme suivant :

$$
\begin{gathered}
\max _{x \in \mathbb{R}^{p}}\left\{x^{\prime} A x\right\} \\
\text { s. c. }\|x\|_{2}=1,
\end{gathered}
$$

où $A=T^{-1} M^{\prime} M$ désigne la matrice de variance-covariance de $M$ de dimension $(p, p)$, $M^{\prime}$ la matrice transposée de $M, T$ la taille de l'échantillon, et $\|x\|_{2}$ désigne la norme deux du vecteur $x$.

La première composante est donc obtenue en maximisant la variance empirique des données projetées sous une contrainte d’identification liée à la norme. La projection des données sur cette composante permet en effet d’obtenir un facteur, noté ici $F$, de dimension $(T, 1)$, avec $F=M x$, dont la variance appelée valeur propre, égale à $\lambda=$ $T^{-1} F^{\prime} F$ est le critère d'optimisation du programme (A.4.1). Dans les travaux portant sur la construction d'indices agrégés de risque systémique, l’indice est généralement associé au facteur F (Moreno et Peña [2013] ; Giglio et al. [2016]).

Le programme d’optimisation précédent, qui permet d’obtenir la première composante ainsi que le facteur dominant, a une représentation équivalente en termes de régression linéaire comme montré par Tibshirani [1996] et par Zou et al. [2006]). Ces derniers proposent de modifier cette régression linéaire dans le but d'obtenir une composante principale dite parcimonieuse (Sparse en anglais) ${ }^{26}$. En effet, si on note $x^{S}$ cette composante, celle-ci est égale à :

$$
x^{s}=\frac{\hat{\beta}^{s}}{\left\|\hat{\beta}^{s}\right\|_{2}},
$$

${ }^{26}$ Cf. également Kouontchou et al. [2017] pour plus de détails sur la méthodologie. 
où $\hat{\beta}^{s}$ est la solution de la régression contrainte (ou pénalisée) suivante :

$$
\begin{gathered}
F=M \beta^{s}+U \\
\text { s.c. } P\left(\beta^{s}\right) \equiv\left\|\beta^{s}\right\|_{1}=\sum_{j=1}^{p}\left|\beta_{j}^{s}\right| \leq \delta .
\end{gathered}
$$

A titre d'illustration, le graphique A.4.1 présente ainsi parmi les évolutions temporelles des «cas extrêmes » des 16 facteurs (ou indices agrégés) : ceux issus des composantes parcimonieuses du tableau 2 liées aux deux cas extrêmes (pour des paramètres de troncature égaux à $\delta=1,000$ et $\delta=2,388$ ) ; il montre ainsi l'intérêt de la méthodologie.

\section{Graphique A.4.1 : Dynamique des indices agrégés (cas limites)}

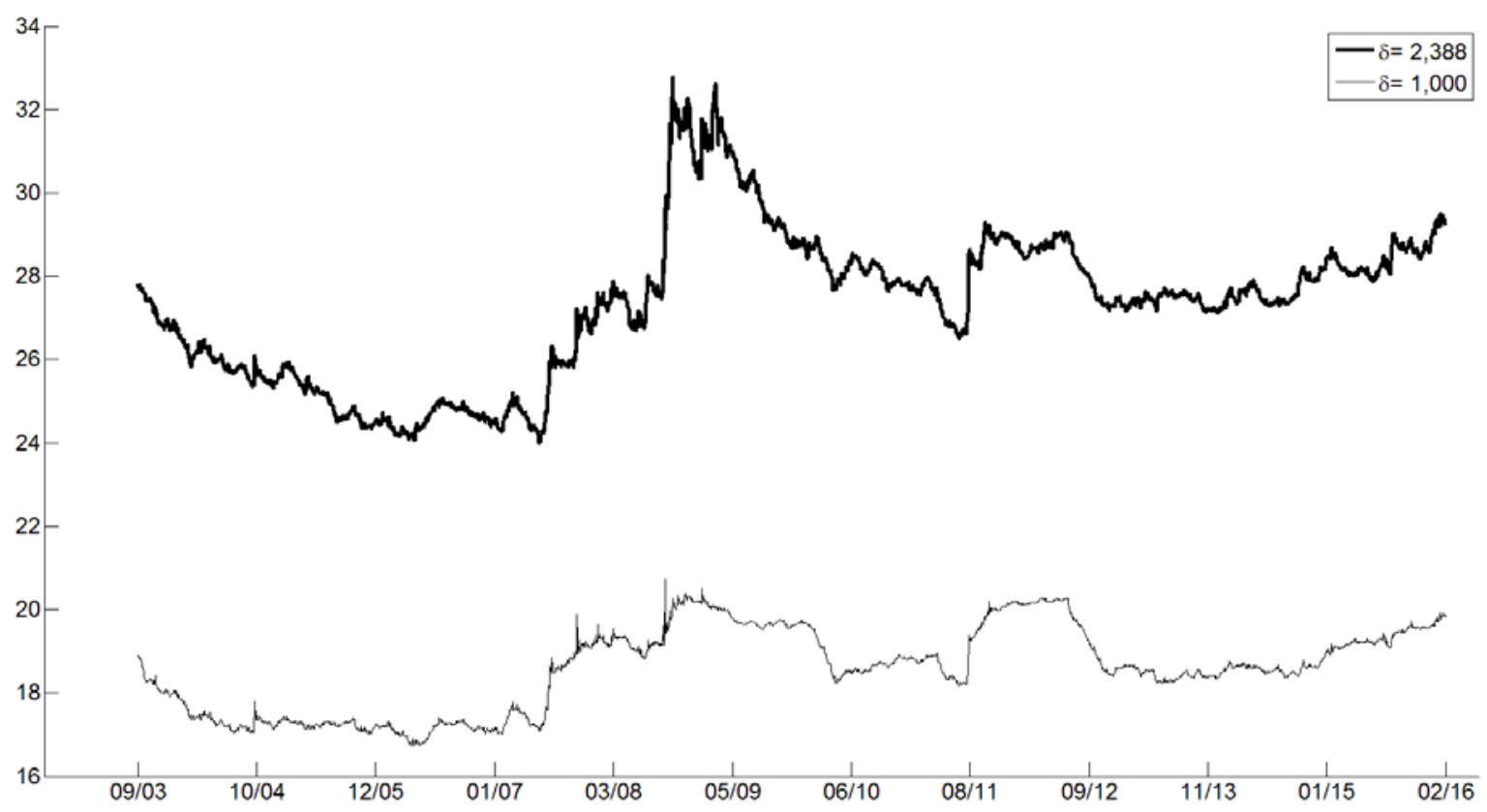

Source : Bloomberg, données journalières du 02/09/2003 au 26/02/2016 ; calculs des auteurs. Sont représentées les deux séries concurrentes de l'indice agrégé des risques systémiques ( $\delta=1,000$ et $\delta=2,388$ ).

En effet, bien que la dynamique temporelle des indices agrégés soit similaire, la variabilité temporelle est sensiblement différente. L'indice le plus stable correspond bien évidemment au cas $\delta=1,000$ avec une variance égale à 1 . Cet indice est uniquement 
déterminé par le Spillover Index de Diebold et Yilmaz [2009]. ${ }^{27}$ L'indice agrégé le plus volatile est obtenu pour $\delta=2,388$ et il est égal au facteur dominant d'une ACP classique. Les autres indices agrégés non représentés affichent des variances comprises entre ces deux valeurs. On retrouve ainsi avec cette analyse l'objectif principal de l'ACPP, à savoir la stabilisation temporelle des facteurs, ici nos indices agrégés. Cependant et comme déjà mentionné, cette stabilisation s'obtient via un arbitrage biais-variance, et il induit donc une baisse de la représentativité (variance expliquée du facteur).

\section{Annexe A.5. Du MEDAF traditionnel au MEDAF étendu au risque systémique}

Nous reprenons la théorie du Modèle d'Equilibre des Actifs Financiers (MEDAF) proposé par Sharpe [1964], Lintner [1965] et Mossin [1966], et son extension à trois facteurs de Fama et French [1993] prenant en compte certaines anomalies dans la valorisation des prix des actifs (Cf. Fama et French [1996]). Puis, nous introduisons ensuite un facteur lié au risque systémique dans le MEDAF étendu au modèle à trois facteurs de Fama-French [1993], au moyen d'un facteur additionnel correspondant à l’indice de risque systémique optimal construit dans la section précédente.

Dans la relation d'équilibre proposée par Sharpe [1964], les rendements des actifs peuvent être expliqués par les rendements du portefeuille de marché tels que :

$$
r_{i}=r_{f}+\beta_{i}\left(r_{M}-r_{f}\right)+\varepsilon_{i}
$$

où $r_{i}$ et $r_{M}$ sont respectivement les rendements du titre $i$ et les rendements du portefeuille de marché, $r_{f}$ est le taux sans risque supposé constant, et $\varepsilon_{i}$ le terme résiduel de moyenne nulle et $\beta_{i}$ l'exposition de l'actif au risque systématique définie comme :

$$
\beta_{i}=\frac{\operatorname{Cov}\left(r_{i}, r_{M}\right)}{\sigma^{2}\left(r_{M}\right)}
$$

${ }^{27}$ L'égalité à 1 de la variance provient de la standardisation des données préalable à l'ACPP. 
Cette relation permet de déterminer, sous certaines conditions, l'espérance de rendement du titre $i$ en fonction de l'exposition de l'actif au risque systématique telle que :

$$
E\left(r_{i}\right)=E\left(r_{f}\right)+\beta_{i}\left[E\left(r_{M}-r_{f}\right)\right]
$$

où $E\left(r_{i}\right)$ et $E\left(r_{M}\right)$ sont respectivement les espérances des rendements du titre $i$ et des rendements du marché, $E\left(r_{f}\right)$ reste égale au taux sans risque.

Ce qui nous amène maintenant à la version canonique du MEDAF étendue au modèle à trois facteurs de Fama-French [1993] dont la représentation est la suivante :

$$
E\left(r_{i}\right)=E\left(r_{f}\right)+\beta_{i}\left[E\left(r_{M}-r_{f}\right)\right]+\gamma_{i}\left[E\left(r_{S M B}-r_{f}\right)\right]+\theta_{i}\left[E\left(r_{H M L}-r_{f}\right)\right]
$$

où $r_{S M B}$ représente les rendements du facteur Small Minus Big (noté SMB), i.e. l'écart de rentabilité entre un portefeuille de titre à faible capitalisation (Small) et un portefeuille de titre à forte capitalisation (Large), et où $r_{H M L}$ représente le facteur High Minus Low (noté $H M L)$, i.e. l'écart de rentabilité entre un portefeuille composé de titres avec un ratio Book Equity sur Market Value élevé et un portefeuille pour lequel ce ratio est faible.

Ainsi, des portefeuilles composés d'entreprises avec des rendements de long terme faibles (appelés Losers), auront tendance à avoir un ratio Book Equity sur Market Value élevé et donc un coefficient devant la prime de risque sur le facteur $H M L$ positif. Inversement, des portefeuilles avec des rendements de long terme plutôt élevés (Winners), auront tendance à avoir un ratio Book Equity sur Market Value faible, et ainsi un coefficient devant la prime de risque sur le facteur $H M L$ négatif.

Pour les rendements du facteur $S M B$, des portefeuilles composés d'entreprises avec des rendements élevés auront tendance à avoir un coefficient positif par rapport aux portefeuilles composés de firmes ayant des rendements faibles, qui auront en conséquence un coefficient négatif caractérisant le niveau de la prime de risque sur le facteur SMB.

Nous ajoutons par la suite l'indice de risque systémique optimal évoqué à la section précédente (ISRM), comme facteur additionnel dans le modèle à trois-facteurs de Fama et French [1993]. Nous obtenons ainsi un modèle à quatre facteurs - modèle (I) ci-dessous - en comparaison du modèle de Fama et French [1993] - modèle (II) ci-dessous, tels que : 
$\left\{\begin{array}{l}r_{i, t}=\alpha_{i}^{(I)}+\beta_{i}^{(I)} r_{M, t}+\theta_{i}^{(I)} r_{S M B, t}+\gamma_{i}^{(I)} r_{H M L, t}+\varepsilon_{i, t}^{(I)} \\ r_{i, t}=\alpha_{i}^{(I I)}+\beta_{i}^{(I I)} r_{M, t}+\theta_{i}^{(I I)} r_{S M B, t}+\gamma_{i}^{(I I)} r_{H M L, t}+\varphi_{i}^{(I I)} r_{I S R M, t}+\varepsilon_{i, t}^{(I I)}\end{array}\right.$

où les facteurs au temps $t$, sont les rendements du marché $r_{M, t}$, les rendements du facteur $S M B$ notés $r_{S M B, t}$, ceux du facteur $H M L$ notés $r_{H M L, t}$ et où le facteur de risque systémique noté $r_{I S R M, t}$ représentant les rendements de l'indice optimal est le quatrième facteur.

Une fois que nous avons écrit la relation de l'évaluation des actifs dans leur forme la plus simple, en insistant sur la relation linéaire entre le rendement et les primes, nous devons maintenant faire face à d'autres particularités des marchés financiers.

En particulier, nous devons d'abord prendre en compte dans la technique d'estimation les potentielles interrelations entre les rendements des actifs. Nous utilisons la méthode SURE de Zellner [1962] (pour Seemingly Unrelated Regressions Estimation) du modèle proposé (Cf. Kraus et Litzenberg, [1976] ; Barone-Adesi et al., [2003] ; Galagedera et Maharaj, [2008]). Nous considérerons ainsi un système de $N$ équations simultanées, dont la i-ième équation est définie dans l'équation (A.5.4).

De façon plus compacte, les vecteurs des différentes rentabilités peuvent être empilés dans un vecteur $\boldsymbol{r}$ de dimension $N T$, avec un même agencement correspondant pour les termes d'erreur, les vecteurs de coefficients, et les régresseurs ; ainsi, nous avons :

$$
\boldsymbol{r}=\left[\begin{array}{c}
r_{1} \\
r_{2} \\
\vdots \\
r_{N}
\end{array}\right], \varepsilon=\left[\begin{array}{c}
\varepsilon_{1} \\
\varepsilon_{2} \\
\vdots \\
\varepsilon_{N}
\end{array}\right], F=\left[\begin{array}{cccc}
F_{1} & 0 & \cdots & 0 \\
0 & F_{2} & \cdots & 0 \\
\vdots & \vdots & & \vdots \\
0 & 0 & \cdots & F_{K+1}
\end{array}\right]
$$

avec $\boldsymbol{r}$ le vecteur des rendements de taille $(N T \times 1)$ où $\boldsymbol{r}_{\boldsymbol{i}}=\left(r_{i, t=1}, \ldots, r_{i, t=T}\right)^{\prime}$ pour $i=$ $(1, \ldots, N)$, $\boldsymbol{\varepsilon}$ le vecteur des termes d'erreur de taille $(N T \times 1)$ où $\boldsymbol{\varepsilon}_{\boldsymbol{i}}=\left(\varepsilon_{i, t=1}, \ldots, \varepsilon_{i, t=T}\right)^{\prime}$ et $\boldsymbol{F}$ la matrice de taille $[N T \times(K+1) T]$ des facteurs définis comme suit : 


$$
\left\{\begin{array}{l}
\boldsymbol{F}_{\mathbf{1}}=(1,1 \ldots, 1)^{\prime} \\
\boldsymbol{F}_{\mathbf{2}}=\left(r_{M, t=1}, \ldots, r_{M, t=T}\right)^{\prime} \\
\boldsymbol{F}_{\mathbf{3}}=\left(r_{S M B, t=1}, \ldots, r_{S M B, t=T}\right)^{\prime} \\
\boldsymbol{F}_{\mathbf{4}}=\left(r_{H M L, t=1}, \ldots, r_{H M L, t=T}\right)^{\prime} \\
\boldsymbol{F}_{\mathbf{5}}=\left(r_{I S R M, t=1}, \ldots, r_{I S R M, t=T}\right)^{\prime} .
\end{array}\right.
$$

Ce qui nous conduit à l'équation suivante :

$$
\boldsymbol{r}=\boldsymbol{F} \boldsymbol{\Lambda}+\boldsymbol{\varepsilon}
$$

où $\boldsymbol{\Lambda}=\left(\boldsymbol{\Lambda}_{1}, \boldsymbol{\Lambda}_{2}, \ldots, \boldsymbol{\Lambda}_{\boldsymbol{N}}\right)$ de taille $[(K+1) T \times 1]$ avec $\boldsymbol{\Lambda}_{\boldsymbol{i}}=\left(\alpha_{i}, \beta_{i}, \theta_{i}, \gamma_{i}, \varphi_{i}\right)^{\prime}$ le vecteur des paramètres de taille $[1 \times(K+1)]$. Ce système est de fait identique à celui proposé par Zellner [1962].

Ainsi, sachant que $\varepsilon_{i, t}$ est le $i$-ème terme d'erreur pour l'actif $i$ à la période $t$, l'hypothèse de la corrélation contemporaine des erreurs, mais d'absence de corrélation sur l'ensemble de la période, implique que la matrice de variance-covariance dans le système et dénotée $\boldsymbol{V}(\boldsymbol{\varepsilon})$ est :

$$
V(\varepsilon)=\Sigma \otimes I_{N}
$$

où :

$$
\boldsymbol{\Sigma}=\left[\begin{array}{cccc}
\sigma_{11} & \sigma_{12} & \cdots & \sigma_{1 T} \\
\sigma_{21} & \sigma_{22} & \cdots & \sigma_{2 T} \\
\vdots & \vdots & & \vdots \\
\sigma_{T 1} & \sigma_{T 2} & \cdots & \sigma_{T T}
\end{array}\right],
$$

avec $\boldsymbol{I}_{\boldsymbol{N}}$ la matrice identité de dimension $N, \boldsymbol{\Sigma}$ la matrice de variance-covariance des résidus de taille $(T \times T)$ où $\sigma_{i i}=E\left(\boldsymbol{\varepsilon}_{\boldsymbol{i}} \boldsymbol{\varepsilon}_{\boldsymbol{i}}\right)$ est la variance de $\boldsymbol{\varepsilon}_{\boldsymbol{i}}$ et $\sigma_{i j}=E\left(\boldsymbol{\varepsilon}_{\boldsymbol{i}} \boldsymbol{\varepsilon}_{\boldsymbol{j}}\right)$ pour $i \neq$ $j$, avec $\otimes$ la notation du produit de Kronecker indiquant que chaque élément de $\boldsymbol{\Sigma}$ est multiplié par $\boldsymbol{I}_{\boldsymbol{N}}$.

Avec les notations précédentes, l'estimateur des Moindres Carrés Ordinaires (MCO) classique pour le vecteur $\boldsymbol{\Lambda}$ s'écrit ainsi : 


$$
\widehat{\boldsymbol{\Lambda}}_{M C O}=\left(\boldsymbol{F}^{\prime} \boldsymbol{F}\right)^{-1} \boldsymbol{F}^{\prime} \boldsymbol{r},
$$

et pour les Moindres Carrés Généralisés (MCG - en supposant que la matrice $\boldsymbol{\Sigma}$ est connue), celui-ci s'écrit :

$$
\widehat{\boldsymbol{\Lambda}}_{M C G}=\left[\boldsymbol{F}^{\prime}(\boldsymbol{\Sigma} \otimes \boldsymbol{I})^{-1} \boldsymbol{F}\right]^{-1} \boldsymbol{F}^{\prime}(\boldsymbol{\Sigma} \otimes \boldsymbol{I})^{-1} \boldsymbol{r} .
$$

Ce système correspond enfin aux MEDAFS lorsque $\boldsymbol{\Lambda}$ correspond à la matrice des sensibilités aux trois facteurs de Fama-French avec une constante auxquels on ajoute le facteur de risque systémique. Les résultats empiriques suivants sont ainsi basés sur le système d'équations (A.5.10), en lien avec la relation linéaire de l'équation (A.5.5), écrite sous forme matricielle dans l'équation (A.5.6).

\section{Annexe A.6. Décomposition du risque et impact sur risque systémique à la Billio} et al. [2017]

Dans cette annexe, nous présentons brièvement un résumé du modèle de représentation des liens entre risque systémique et risque systématique tel qu’illustré dans Billio et al. [2017].

Plus précisément, les auteurs envisagent une relation telle qu'en exprimant le Modèle (I) sous forme matricielle et en prenant en compte les interconnexions entre les actifs, l'équation (A.5.6) se réécrirait en gardant les mêmes notations :

$$
A r=\Lambda F+\varepsilon,
$$

avec $\boldsymbol{A}$ la matrice des relations entre les titres de dimension $(N \times N)$ telle que $\boldsymbol{A}=\boldsymbol{I}_{\boldsymbol{N}}-$ $\rho \boldsymbol{W}$ où $\boldsymbol{I}_{\boldsymbol{N}}$ représente la matrice identité de dimension $(N \times N), \rho$ un scalaire représentant l'impact global que les autres actifs ont sur l'actif $i$ (Billio et al. [2017] estiment différentes valeurs pour $\rho$ en fonction du secteur financier étudié) et $\boldsymbol{W}$ représente la 
matrice spatiale de dimension $(N \times N)$ des connections, dont les éléments $\boldsymbol{W}_{i, j}$ représentent les liens entre les titres $i$ et $j$.

En divisant chaque terme de l'équation précédente par la matrice $\boldsymbol{A}$, celle-ci peut se réécrire comme suit :

$$
r=A^{-1} \Lambda F+A^{-1} \varepsilon
$$

En posant $\boldsymbol{\Lambda}_{s}=\boldsymbol{A}^{-\mathbf{1}} \boldsymbol{\Lambda}$, le vecteur de paramètres augmentés du risque lié à l'interconnexion, la relation devient :

$$
r=\Lambda_{S} F+A^{-1} \varepsilon
$$

Cette dernière relation permet de tenir compte du risque systémique. Par la suite, Billio et al. [2017] montrent que l'équation de décomposition de la variance $\boldsymbol{\Sigma}_{\boldsymbol{r}}=\boldsymbol{V}(\boldsymbol{r})$ dans ce cas est donnée par :

$$
\begin{aligned}
& \Sigma_{r}=\Lambda_{s} \Sigma_{F} \Lambda_{s}^{\prime}+A^{-1} \boldsymbol{\Omega}\left(A^{-1}\right)^{\prime} \\
& \quad=\Lambda_{s} \Sigma_{F} \Lambda_{s}^{\prime}+A^{-1} \boldsymbol{\Omega}\left(A^{-1}\right)^{\prime}+\Lambda \Sigma_{F} \Lambda^{\prime}-\Lambda \Sigma_{F} \Lambda^{\prime}+\Omega-\Omega \\
& \quad=\left\{\Lambda \Sigma_{F} \Lambda^{\prime}\right\}+\{\Omega\}+\left\{\Lambda_{s} \Sigma_{F} \Lambda_{s}^{\prime}-\Lambda \Sigma_{F} \Lambda^{\prime}\right\}+\left\{A^{-1} \boldsymbol{\Omega}\left(A^{-1}\right)^{\prime}-\boldsymbol{\Omega}\right\}
\end{aligned}
$$

avec $\boldsymbol{\Sigma}_{\boldsymbol{F}}=\boldsymbol{V}(\boldsymbol{F})$ la matrice de variance-covariance des facteurs et $\boldsymbol{\Omega}=\boldsymbol{V}(\boldsymbol{\varepsilon})=\boldsymbol{\Sigma}_{\boldsymbol{\varepsilon}} \otimes \boldsymbol{I}_{\boldsymbol{N}}$ la matrice de variance-covariance des erreurs telle que l'élément $[\Omega]_{i, j}$ pour $i=j$, représente le risque spécifique du titre $i$ et telle que les autres éléments $[\Omega]_{i, j}=0$ pour tout $i \neq j$.

Au final, le risque global peut être décomposé en quatre sources de risque distinctes :

- les risques liés aux facteurs : $\boldsymbol{\Lambda}_{S} \boldsymbol{\Sigma}_{\boldsymbol{F}} \boldsymbol{\Lambda}_{S}^{\prime}$;

- les risques spécifiques liés aux titres : $\mathbf{\Omega}$;

- l'impact du risque systémique sur les facteurs : $\boldsymbol{\Lambda}_{S} \boldsymbol{\Sigma}_{\boldsymbol{F}} \boldsymbol{\Lambda}_{S}^{\prime}-\boldsymbol{\Lambda} \boldsymbol{\Sigma}_{\boldsymbol{F}} \boldsymbol{\Lambda}^{\prime}$;

- l'impact du risque systémique sur les risques spécifiques : $\boldsymbol{A}^{\mathbf{- 1}} \boldsymbol{\Omega}\left(\boldsymbol{A}^{-\mathbf{1}}\right)^{\prime}-\boldsymbol{\Omega}$.

Ainsi, en additionnant les impacts du risque systémique sur les risques systématiques et spécifiques, on obtient la contribution totale du réseau (et donc du risque systémique) au risque total, qui n'est autre que la somme de ces quatre composantes de risque. 
Dans le cadre de notre analyse, dans laquelle le nombre de connections interinstitutions intervient dans la construction de l'indice (au travers de la prise en compte du DCI de Billio et al. [2012]) nous retrouvons cet effet croisé entre risque systématique et risque systémique, qui représente le risque systémique comme une composante extrême du risque systématique normal.

Annexe A.7. Evolutions des coefficients de sensibilité au risque systématique et au risque systémique pour JPM

La recherche d'un classement stable est tout à fait légitime du point de vue du régulateur. Le graphique A.7.1 montre l'évolution des sensibilités aux risques systématique et systémique pour JPM. Il est reconnu que le beta d'un actif est relativement stable dans le temps. On constate sur ce graphique que la sensibilité au risque systémique a la même propriété de stabilité.

Graphique A.7.1 : Evolutions des coefficients de sensibilité au risque systématique et au risque systémique pour JPM

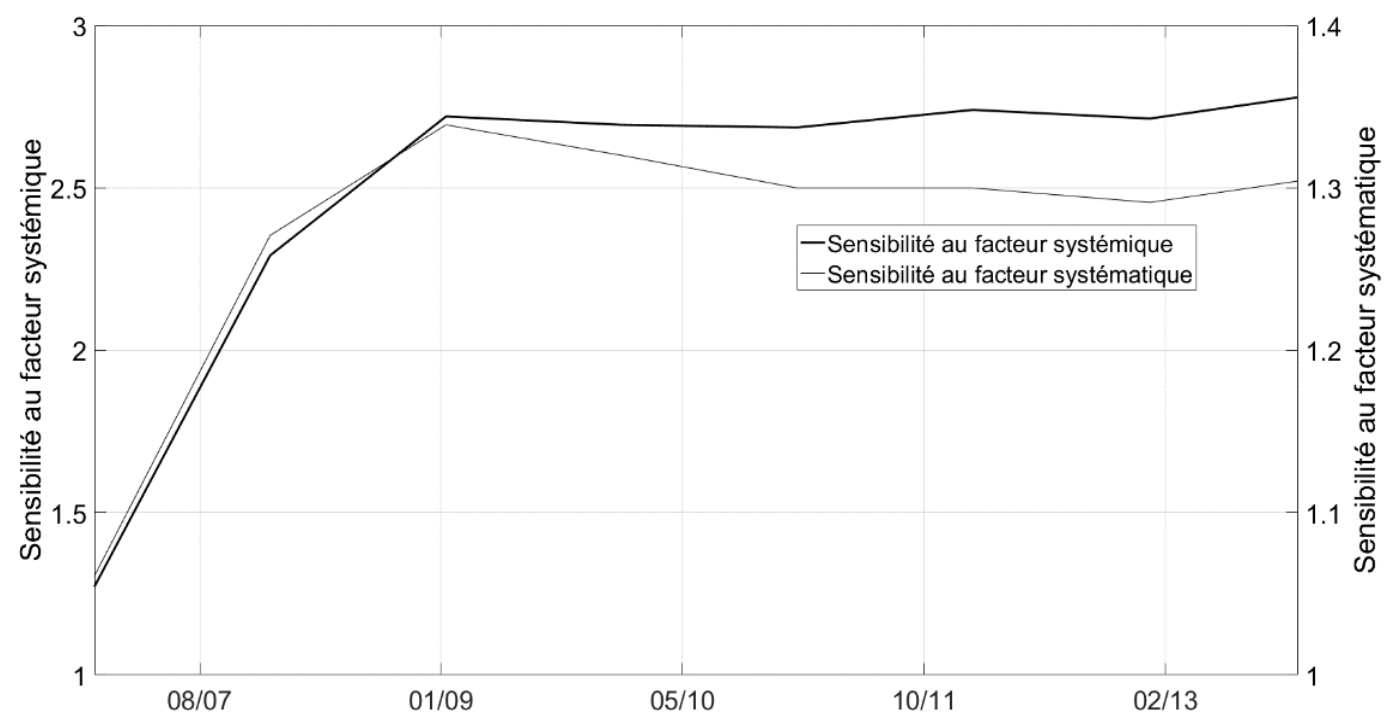

Source : Bloomberg, séries journalières du 03/09/2003 au 24/06/2014. Sont représentées les sensibilités aux facteurs de risque systématique (trait noir) et systémique (trait gris clair) en valeurs absolues pour JPM.

Ainsi, deux IFIS qui auraient des sensibilités au risque systémique très proches, pourraient effectivement changer de rang dans le classement, mais ce changement pourrait être considéré comme anecdotique. L’ampleur et la significativité du risque systémique doivent être prises en compte conjointement. 